\title{
Asymptotically almost periodic mild solutions to a class of Weyl-like fractional difference equations
}

\author{
Junfei Cao' ${ }^{1}$, Bessem Samet ${ }^{2}$ and Yong Zhou ${ }^{3,4^{*}}$ (i)
}

\begin{tabular}{l}
\hline${ }^{*}$ Correspondence: \\
yzhou@xtu.edu.cn \\
${ }^{3}$ Faculty of Mathematics and \\
Computational Science, Xiangtan \\
University, Xiangtan, P.R. China \\
${ }^{4}$ Nonlinear Analysis and Applied \\
Mathematics (NAAM) Research \\
Group, Faculty of Science, King \\
Abdulaziz University, Jeddah, Saudi \\
Arabia \\
Full list of author information is \\
available at the end of the article
\end{tabular}

available at the end of the article

\begin{abstract}
This paper is concentrated on a class of difference equations with a Weyl-like fractional difference in a Banach space $X$ forms like

$$
\triangle^{\alpha} x(n)=A x(n+1)+F(n, x(n)), \quad n \in \mathbb{Z},
$$

where $\alpha \in(0,1)$, the operator $A$ generates a $C_{0}$-semigroup on $X, \triangle^{\alpha}$ denotes the Weyl-like fractional difference operator, $F(n, X): \mathbb{Z} \times X \rightarrow X$ is a nonlinear function. Some existence theorems for asymptotically almost periodic mild solutions to this system are obtained with the nonlinear perturbation $F$ being of Lipschitz type or non-Lipschitz type. The results are a consequence of applications of the Banach contraction mapping theory, the Leray-Schauder alternative theorem, and Matkowski's fixed point theorem. As an application, an example is provided to show the feasibility of the theoretical results.
\end{abstract}

MSC: $39 \mathrm{~A} 14 ; 34 \mathrm{D} 05$

Keywords: Asymptotically almost periodic sequence; Fractional difference equation; Fixed point theorem; Leray-Schauder alternative theorem

\section{Introduction}

Difference equations are an important area of applied mathematics, they appear as the variational equations along the trajectories of dynamical systems with discrete time, and they play an important role in numerical analysis, systems theory, computer science and so on [1-3]. The theory of difference equations is richer than the corresponding theory of differential equations since difference equations are more appropriate than their continuous counterparts in cases when processes evolve in stages.

Difference equation models are a powerful tool in describing natural phenomena and characterizing many physical problems. Due to their numerous application in many fields, difference equation models have attracted increasing interest in recent years, and one of the central topics in the study of difference equations is the investigation of the existence, uniqueness of solutions with almost periodicity and asymptotically almost periodicity. The original notion of discrete almost periodic sequence was proposed by Walther [4, 5] and was further studied by Halanay [6] and Corduneanu [7] related to some aspects of con-

(c) The Author(s) 2019. This article is distributed under the terms of the Creative Commons Attribution 4.0 International License (http://creativecommons.org/licenses/by/4.0/), which permits unrestricted use, distribution, and reproduction in any medium, provided you give appropriate credit to the original author(s) and the source, provide a link to the Creative Commons license, and indicate if changes were made. 
tinuous almost periodic solutions for differential equations. As an important development of the well-known discrete almost periodic sequence, the notion of discrete asymptotically almost periodic sequence, which is based upon the Fréchet concept from [8, 9], was introduced in the work of Fan [10] in 1943. Since then, this concept has been developed in different directions and has been generalized into various situations, the theory and applications of discrete asymptotically almost periodic sequences have attracted increasing attention in recent years. In particular, the investigation of the existence of solutions with asymptotically almost periodicity has become one of the important as well as attractive subjects in the theory as well as applications of difference equations due to both the intensive development of the theorem of difference equations itself and the applications in various sciences such as computer science, chemistry, physics, engineering. Recently, a lot of papers have been published devoted to the investigation of asymptotically almost periodicity of difference equation of various types (see for instance [11-16] and the references therein).

On the other hand, the tools of fractional calculus are found to be of great utility in studying various scientific processes and systems. It has been mainly due to the ability of fractional order operators to describe long-memory effects of underlying processes. In particular, models described by fractional differential equations have gained significant importance and there has been a great interest in developing the theory and applications of fractional differential equations. For examples and details, we refer the reader to a series of recent research articles [17-21] and the references therein. In recent years, difference equations of fractional order have attracted increasing interest of many researchers. Besides their interest in terms of theory, the study of fractional difference equations has great importance as regards applications. The study of modeling with fractional difference equations began with the work of Atici and Sengül [22], and then Goodrich studied the existence, uniqueness and positivity [23, 24] as well as monotonicity properties [25] of solutions to the discrete boundary value problem of fractional order. More recently, Wu and Baleanu [26] studied the discrete fractional logistic map and its chaos. The abstract fractional difference equations began to be studied by Lizama [27]. He studied the existence as well as stability of fractional difference equations forms like

$$
\left\{\begin{array}{l}
{ }^{C} \triangle^{\alpha} x(n)=A x(n+1), \quad n \in N, \\
x(0)=x_{0} \in X
\end{array}\right.
$$

where $\alpha \in(0,1),{ }^{C} \triangle^{\alpha}$ is the Caputo-like fractional difference operator, and $A$ is a closed linear operator defined on a Banach space $X$. Especially in [28], Abadias and Lizama consider a nonlinear partial difference-differential equations in a Banach space $X$ forms like

$$
\triangle^{\alpha} x(n)=A x(n+1)+F(n, x(n)), \quad n \in \mathbb{Z},
$$

where $\alpha \in(0,1)$, the operator $A$ generates a $C_{0}$-semigroup defined on $X, \triangle^{\alpha}$ denotes the Weyl-like fractional difference operator, $F(n, x): \mathbb{Z} \times X \rightarrow X$ is a nonlinear function. By using the operator theoretical method and the Banach fixed point theorem, they prove the existence and uniqueness of almost automorphic solutions to Eq. (1.1).

However, it should be mentioned that literature about the existence of solutions with asymptotically almost periodicity to fractional difference equations is scarce. Motivated 
by the above facts, in this paper we give some existence theorems for mild solutions with asymptotically almost periodicity to the fractional difference equation (1.1) with the nonlinear perturbation $F$ is Lipschitz type or non-Lipschitz type. In particular, as an application, we prove the existence and uniqueness of mild solutions with asymptotically almost periodicity to the fractional difference equations in a Banach space $X$ of forms like

$$
\triangle^{\alpha} x(n)=A x(n+1)+\frac{\mu g(n)[1+x(n)]}{1+\sup \{\|x(n)\|: n \in \mathbb{Z}\}}, \quad n \in \mathbb{Z},
$$

where $\alpha \in(0,1)$, the operator $A$ generates a $C_{0}$-semigroup defined on $X, g(n): \mathbb{Z} \rightarrow X$ is a sequence which is asymptotically almost periodic, and $\mu>0$ is a parameter.

An outline of this article is as follows. We introduce some basic concepts and recall some preliminaries in Sect. 2. Section 3 is concerned with the existence of mild solutions with asymptotically almost periodicity of the nonhomogeneous linear difference equations corresponding to Eq. (1.1). In Sect. 4, we give some existence theorems for mild solutions with asymptotically almost periodicity to the nonlinear equation (1.1). The last section deal with an example to validate the applications of our theoretical results.

\section{Preliminaries}

In this section, we introduce some basic concepts and recall some preliminaries.

In this paper, let $\mathbb{N}, \mathbb{Z}, \mathbb{Z}^{+}, \mathbb{R}, \mathbb{R}^{+}, \mathbb{C}$ be the sets of all natural numbers, integral numbers, positive integral numbers, real numbers, positive real numbers, complex numbers, respectively. For a Banach spaces $(X,\|\cdot\|)$, let $B_{\rho}(X)=\{x \in X:\|x\| \leq \rho\} . s(\mathbb{Z}, X)$ is a set consisting of sequences $f: \mathbb{Z} \rightarrow X . l^{\infty}(\mathbb{Z}, X)$ is a set consisting of sequences

$$
l^{\infty}(\mathbb{Z}, X):=\{f: \mathbb{Z} \rightarrow X \mid f \text { is bounded on } \mathbb{Z}\}
$$

The space $l^{\infty}(\mathbb{Z}, X)$ is a Banach space under the norm

$$
\|x\|_{d}:=\sup _{n \in \mathbb{Z}}\|x(n)\| .
$$

$A A P_{0}(\mathbb{Z}, X)$ is a set consisting of sequences

$$
A A P_{0}(\mathbb{Z}, X):=\left\{f(n) \in l^{\infty}(\mathbb{Z}, X) \mid \lim _{|n| \rightarrow+\infty}\|f(n)\|=0\right\} .
$$

$A A P_{0}(\mathbb{Z}, X)$ is also a Banach space under the norm $\|x\|_{d} . l(\mathbb{Z}, X)$ is a set consisting of sequences

$$
l(\mathbb{Z}, X):=\left\{f: \mathbb{Z} \rightarrow X \mid\|f\|_{l}=\sum_{n=-\infty}^{+\infty}\|f(n)\|<+\infty\right\} .
$$

Moreover, when $X=\mathbb{R}$, we write $l(\mathbb{Z})$ for short. $l_{\rho}(\mathbb{Z}, X)$ is a set consisting of sequences

$$
\begin{aligned}
l_{\rho}(\mathbb{Z}, X):= & \left\{f: \mathbb{Z} \rightarrow X \mid\|f\|_{l_{\rho}}=\sum_{n=-\infty}^{+\infty}\|f(n)\| \rho(n)<+\infty,\right. \\
& \left.\rho: \mathbb{Z} \rightarrow \mathbb{R}^{+} \text {is a positive sequence weight }\right\} .
\end{aligned}
$$


Let $\left(Y,\|\cdot\|_{Y}\right)$ be another Banach space, $l^{\infty}(\mathbb{Z} \times Y, X)$ is a set consisting of functions

$$
\begin{aligned}
l^{\infty}(\mathbb{Z} \times Y, X):= & \{G: \mathbb{Z} \times Y \rightarrow X \mid G \text { is bounded on } \mathbb{Z} \times Y \text { and } \\
& G(n, \cdot) \text { is continuous on } Y \text { for each fixed } n \in \mathbb{Z}\} .
\end{aligned}
$$

The space $l^{\infty}(\mathbb{Z} \times Y, X)$ is a Banach space under the norm

$$
\|G\|:=\sup _{n \in \mathbb{Z}, x \in Y}\|G(n, x)\| .
$$

$A A P_{0}(\mathbb{Z} \times Y, X)$ is a set consisting of functions

$$
\begin{aligned}
& A A P_{0}(\mathbb{Z} \times Y, X):=\left\{\left.G(n, x) \in l^{\infty}(\mathbb{Z} \times Y, X)\right|_{|n| \rightarrow+\infty}\|G(n, x)\|=0\right. \\
&\text { uniformly for } x \in Y\}, \\
& U C(\mathbb{Z} \times Y, X)=\{G: \mathbb{Z} \times Y \rightarrow X \mid \forall \varepsilon>0, \exists \delta>0 \text {, s.t. }\|G(n, x)-G(n, y)\| \leq \varepsilon \\
&\left.\forall n \in \mathbb{Z} x, y \in Y \text { with }\|x-y\|_{Y} \leq \delta\right\}, \\
& U C_{n}(\mathbb{Z} \times Y, X)=\left\{G: \mathbb{Z} \times Y \rightarrow X \mid \forall \varepsilon>0, \exists \delta>0 \text {, s.t. }\|G(n, x)-G(n, y)\| \leq L_{f}(n) \varepsilon\right. \\
&\left.\forall n \in \mathbb{Z} x, y \in Y \text { with }\|x-y\|_{Y} \leq \delta, L_{f} \in l(\mathbb{Z})\right\} .
\end{aligned}
$$

Let $\mathbb{L}(X, Y)$ be the collection of all bounded linear operators from $X$ to $Y$. Under the uniform operator topology

$$
\|\Upsilon\|_{\mathbb{L}(X, Y)}:=\sup \left\{\|\Upsilon f\|_{Y}: f \in X,\|f\|=1\right\}
$$

we denote $\mathbb{L}(X)=\mathbb{L}(X, X)$. For $A \in \mathbb{L}(X)$, let $\rho(A)$ be the resolvent of $A$ and $D(A)$ be the domain of $A$.

Firstly, we recall the definitions and related properties on discrete almost periodic sequences as well as discrete asymptotically almost periodic sequences.

Following Bohr, Walther has formulated the notion of discrete almost periodic sequence.

Definition 2.1 (Walther [4, 5], Corduneanu [29]) Let $\{f(n)\}_{n \in \mathbb{Z}}$ be a discrete sequence with values in $X$. If for each $\varepsilon>0$, the collection

$$
T(f, \varepsilon):=\{k \in \mathbb{Z}:\|f(n+k)-f(n)\|<\varepsilon \text { for every } n \in \mathbb{Z}\}
$$

is relatively dense in $\mathbb{Z}$, that is, for any $\varepsilon>0$, there is an integer $N=N(\varepsilon)>0$, such that there exists at least one integer $k \in \Delta$, where $\Delta$ is any collection consisting of $N$ consecutive integers, satisfying

$$
\|f(n+k)-f(n)\|<\varepsilon, \quad n \in \mathbb{Z}
$$

then $\{f(n)\}_{n \in \mathbb{Z}}$ is said to be a discrete almost periodic sequence. 
The integer $k \in T(k, \varepsilon)$, with the property in Definition 2.1, is said to be an $\varepsilon$-translation number of the sequence $\{f(n)\}_{n \in \mathbb{Z}}$.

By $A P(\mathbb{Z}, X)$ we denote the collection of such sequences.

The following notion of a normal process is needed to formulate an important property of the discrete almost periodic processes.

Definition 2.2 (Corduneanu [29], Zhang, Liu and Gopalsamy [11]) A discrete sequence $\{f(n)\}_{n \in \mathbb{Z}}$ is called a normal process, if for any sequence $\{\alpha(k)\} \in \mathbb{Z}$, there is a subsequence $\{\beta(k)\} \subset\{\alpha(k)\}$, for which $\{f(n+\beta(k))\}$ converges uniformly with respect to $n \in \mathbb{Z}$, as $k \rightarrow \infty$. That is to say, for any $\varepsilon>0$, there exist an integer $K(\varepsilon)>0$ and a discrete process $\{\bar{f}(n)\}_{n \in \mathbb{Z}}$ such that

$$
\|f(n+\beta(k))-\bar{f}(n)\|<\varepsilon \quad \text { for } k \geq K(\varepsilon), n \in \mathbb{Z}
$$

Lemma 2.1 (Corduneanu [29]) A discrete process is almost periodic if and only if it is normal.

Lemma 2.2 (Corduneanu [29], Zhang [30, 31])

(I) For any almost periodic sequence $\{f(n)\}_{n \in \mathbb{Z}}$, there is a function $g(t), t \in \mathbb{R}$ which is almost periodic satisfying $g(n)=f(n)$ for $n \in \mathbb{Z}$.

(II) For any almost periodic function $g(t), t \in \mathbb{R},\{g(n)\}_{n \in \mathbb{Z}}$ is an almost periodic sequence.

Remark 2.1 (Campo, Pinto and Vidal [14]) The discretization of a periodic functions may not lead to a periodic sequences. For instance, $\{\cos (k)\}, k=1,2,3, \ldots$, is not a periodic sequence, it is an almost periodic sequence.

Lemma 2.3 (Corduneanu [29], Zhang [30, 31]) $\{f(n)\}_{n \in \mathbb{Z}}$ is bounded if $\{f(n)\}_{n \in \mathbb{Z}}$ is an almost periodic sequence.

Lemma 2.4 (Corduneanu [29], Long and Pan [32]) Under the norm $\|\cdot\|_{d}, A P(\mathbb{Z}, X)$ forms a Banach space.

Definition 2.3 (Abadias and Lizama [28]) An operator-valued sequence $\{\Upsilon(n)\}_{n \in \mathbb{N}} \subset$ $\mathbb{L}(X)$ is summable if

$$
\|\Upsilon\|_{1}:=\sum_{n=0}^{+\infty}\|\Upsilon(n)\|_{\mathbb{L}(X)}<\infty
$$

The following lemma which comes from Gohberg and Feldman [33] is the essential property to study almost periodicity and asymptotic almost periodicity of difference equations.

Lemma 2.5 (Gohberg and Feldman [33]) Assume $\{\Upsilon(n)\}_{n \in \mathbb{N}}$ is a summable sequence. Then, for any discrete sequence $\{f(n)\}_{n \in \mathbb{Z}}$ which is almost periodic, the sequence $\{g(n)\}_{n \in \mathbb{Z}}$ 
defined by

$$
g(n)=\sum_{k=0}^{+\infty} \Upsilon(k) f(n-k), \quad n \in \mathbb{Z}
$$

is also an almost periodic sequence.

Definition 2.4 (Song [13]) Let $G: \mathbb{Z} \times Y \rightarrow X$ and $\Omega$ be any compact set in $Y$. If for any $\varepsilon>0$, the collection

$$
T(G, \varepsilon, \Omega):=\{k \in \mathbb{Z}:\|G(n+k, x)-G(n, x)\|<\varepsilon \text { for each } n \in \mathbb{Z} \text { and } x \in \Omega\}
$$

is relatively dense in $\mathbb{Z}$, that is, for any $\varepsilon>0$, there is an integer $N=N(\varepsilon, \Omega)$ such that there exists at least one integer $k \in \Delta$, where $\Delta$ is any collection consisting of $N$ consecutive integers, satisfying

$$
\|G(n+k, x)-G(n, x)\|<\varepsilon \quad \forall n \in \mathbb{Z}, x \in \Omega,
$$

then $G(n, x)$ is said to be almost periodic in $n \in \mathbb{Z}$ uniformly for $x \in Y$. The integer $k \in$ $T(G, \varepsilon, \Omega)$, with the property in Definition 2.4, is said to be the $\varepsilon$-translation number of $G(n, x)$.

By $A P(\mathbb{Z} \times Y, X)$ we denote the collection of such functions.

Lemma 2.6 (Campo, Pinto and Vidal [14], Song [13]) Let $\Omega$ be any compact set in $Y$ and assume $G \in A P(\mathbb{Z} \times Y, X)$. Then $G(n, \cdot)$ is continuous on $\Omega$ uniformly for $n \in \mathbb{Z}$, that is, for any $\varepsilon>0$, there is a constant $\delta>0$ such that

$$
\|G(n, x)-G(n, y)\|<\varepsilon \quad \forall n \in \mathbb{Z} x, y \in \Omega \text { with }\|x-y\|<\delta,
$$

and $G(\mathbb{Z} \times \Omega)$ is relatively compact in $X$.

Lemma 2.7 (Song [13]) Assume $f \in A P(\mathbb{Z}, X)$. Then, for any integer sequence $\left\{\alpha_{k}\right\}$, there are a subsequence $\left\{\beta_{k}\right\} \subset\left\{\alpha_{k}\right\}$ and a sequence $g: \mathbb{Z} \rightarrow X$ satisfying

$$
f\left(n+\beta_{k}\right) \rightarrow g(n)
$$

uniformly on $\mathbb{Z}$ as $k \rightarrow \infty$. Moreover, $g \in A P(\mathbb{Z}, X)$.

Lemma 2.8 (Song [13]) Assume $G \in A P(\mathbb{Z} \times Y, X)$ and $\Omega$ is any compact set in $Y$. Then, for any integer sequence $\left\{\alpha_{k}\right\}$, there are a subsequence $\left\{\beta_{k}\right\} \subset\left\{\alpha_{k}\right\}$ and a function $H: \mathbb{Z} \times Y \rightarrow$ $X$ satisfying

$$
G\left(n+\beta_{k}, x\right) \rightarrow H(n, x)
$$

uniformly on $\mathbb{Z} \times \Omega$ as $k \rightarrow \infty$. Moreover $H \in A P(\mathbb{Z} \times Y, X)$. 
Lemma 2.9 Assume $G \in A P(\mathbb{Z} \times Y, X)$ and $\Omega$ is any compact set in $Y$. For any $f \in A P(\mathbb{Z}, Y)$ satisfying $f(n) \in \Omega, \forall n \in \mathbb{Z}$, then the sequence $\{G(n, f(n))\}_{n \in \mathbb{Z}}$ is almost periodic.

Proof From $G \in A P(\mathbb{Z} \times Y, X)$ and $f \in A P(\mathbb{Z}, Y)$, together with Lemma 2.7 and Lemma 2.8, it follows that, for any integer sequence $\left\{\alpha_{k}\right\}$, there exist a subsequence $\left\{\beta_{k}\right\} \subset\left\{\alpha_{k}\right\}$ and two functions $H: \mathbb{Z} \times Y \rightarrow X, g: \mathbb{Z} \rightarrow Y$ satisfying

$$
G\left(n+\beta_{k}, x\right) \rightarrow H(n, x)
$$

uniformly on $\mathbb{Z} \times \Omega$ as $k \rightarrow \infty$, and

$$
f\left(n+\beta_{k}\right) \rightarrow g(n)
$$

uniformly on $\mathbb{Z}$ as $k \rightarrow \infty$. Moreover $H \in A P(\mathbb{Z} \times Y, X)$ and $g \in A P(\mathbb{Z}, Y)$. From Lemma 2.6, it follows that $H(n, x)$ is continuous on $\Omega$ uniformly for $n \in \mathbb{Z}$, thus, for any $\varepsilon>0$, one can find a constant $\delta=\delta\left(\frac{\varepsilon}{2}\right)>0$ satisfying

$$
\|H(n, x)-H(n, y)\|<\frac{\varepsilon}{2} \quad \forall n \in \mathbb{Z} x, y \in \Omega \text { with }\|x-y\|_{Y}<\delta .
$$

Moreover, there is a constant $K=K\left(\frac{\varepsilon}{2}\right)$ such that, for all $k>K$,

$$
\begin{aligned}
& \left\|G\left(n+\beta_{k}, x\right)-H(n, x)\right\|<\frac{\varepsilon}{2}, \quad \forall n \in \mathbb{Z} x \in \Omega, \\
& \left\|f\left(n+\beta_{k}\right)-g(n)\right\|<\delta, \quad \forall n \in \mathbb{Z} .
\end{aligned}
$$

On the other hand, since $f\left(n+\beta_{k}\right) \in \Omega, \forall n \in \mathbb{Z}$, for any $k>K$,

$$
\begin{aligned}
& \left\|G\left(n+\beta_{k}, f\left(n+\beta_{k}\right)\right)-H(n, f(n))\right\| \\
& \quad \leq\left\|G\left(n+\beta_{k}, f\left(n+\beta_{k}\right)\right)-H\left(n, f\left(n+\beta_{k}\right)\right)\right\|+\left\|H\left(n, f\left(n+\beta_{k}\right)\right)-H(n, x(n))\right\| \\
& \quad<\frac{\varepsilon}{2}+\frac{\varepsilon}{2}=\varepsilon,
\end{aligned}
$$

which shows $\{G(n, x(n))\}_{n \in \mathbb{Z}}$ is a normal process, then $G(n, x(n))$ is almost periodic which follows from Lemma 2.1.

As an important development of the well-known discrete almost periodic sequence, the notion of discrete asymptotically almost periodic sequence, which is based upon the Fréchet concept from [8,9], was introduced in the literature [10] by Fan.

Definition 2.5 (Fan [10], Song [13]) If a sequence $f(n)=g(n)+h(n)$ with $g(n) \in A P(\mathbb{Z}, X)$ and $h(n) \in A A P_{0}(\mathbb{Z}, X)$, then the sequence $f: \mathbb{Z} \rightarrow X$ is said to be asymptotically almost periodic.

The sequence $\{g(n)\}_{n \in \mathbb{Z}}$ is called the almost periodic component of $\{f(n)\}_{n \in \mathbb{Z}}$, and $\{h(n)\}_{n \in \mathbb{Z}}$ is called the ergodic perturbation of $\{f(n)\}_{n \in \mathbb{Z}}$.

By $A A P(\mathbb{Z}, X)$ we denote the collection of such sequences. 
Lemma 2.10 (Zhang [30, 31])

(I) For any discrete asymptotically almost periodic sequence $\{f(n)\}_{n \in \mathbb{Z}}$, there is a function $g(t), t \in \mathbb{R}$ which is asymptotically almost periodic satisfying $g(n)=f(n)$ for $n \in \mathbb{Z}$.

(II) For any asymptotically almost periodic function $g(t), t \in \mathbb{R},\{g(n)\}_{n \in \mathbb{Z}}$ is a discrete asymptotically almost periodic sequence.

Lemma 2.11 (Zhang [30, 31]) The decomposition of an asymptotically almost periodic sequence $\{f(n)\}_{n \in \mathbb{Z}}$

$$
f(n)=g(n)+h(n)
$$

with $g(n) \in A P(\mathbb{Z}, X)$ and $h(n) \in A A P_{0}(\mathbb{Z}, X)$, is unique.

Lemma 2.12 (Long and Pan [32]) Under the norm $\|\cdot\|_{d}, A A P(\mathbb{Z}, X)$ also forms a Banach space.

Lemma 2.13 Assume $g(n)$ is the almost periodic component of the sequence $f(n) \in$

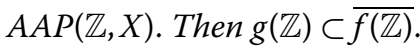

Proof Denote $h(n)=f(n)-g(n)$, the ergodic perturbation of $f(n)$. If $g(\mathbb{Z})$ is not contained in $\overline{f(\mathbb{Z})}$, then there are $\varepsilon_{0}>0$ and $n_{0} \in \mathbb{Z}$ such that

$$
\inf _{n \in \mathbb{Z}}\left\|f(n)-g\left(n_{0}\right)\right\| \geq \varepsilon_{0}
$$

Let us take $k \in T\left(g, \frac{\varepsilon_{0}}{2}\right)$. Then

$$
\begin{aligned}
\left\|h\left(n_{0}+k\right)\right\| & =\left\|f\left(n_{0}+k\right)-g\left(n_{0}+k\right)\right\| \\
& \geq\left\|f\left(n_{0}+k\right)-g\left(n_{0}\right)\right\|-\left\|g\left(n_{0}+k\right)-g\left(n_{0}\right)\right\| \geq \frac{\varepsilon_{0}}{2},
\end{aligned}
$$

which is a contradiction with $h(n) \in A A P_{0}(\mathbb{Z}, X)$.

Definition 2.6 (Campo, Pinto and Vidal [14], Long and Pan [32]) A function $G(n, x)$ : $\mathbb{Z} \times Y \rightarrow X$ is said to be a discrete asymptotically almost periodic function in $n \in \mathbb{Z}$ for each $x \in Y$ if

$$
G(n, x)=H(n, x)+W(n, x)
$$

with $H(n, x) \in A P(\mathbb{Z} \times Y, X)$ and $W(n, x) \in A A P_{0}(\mathbb{Z} \times Y, X)$.

By $A A P(\mathbb{Z} \times Y, X)$ we denote the collection of such functions.

Lemma 2.14 Assume $G(n, x) \in A A P(\mathbb{Z} \times Y, X)$ and $\Omega$ is any compact set in $Y$. Then $G(n, x)$ is bounded on $\mathbb{Z} \times \Omega$.

Proof As $G(n, x) \in A A P(\mathbb{Z} \times Y, X)$, then

$$
G(n, x)=H(n, x)+W(n, x)
$$


with $H(n, x) \in A P(\mathbb{Z} \times Y, X)$ and $W(n, x) \in A A P_{0}(\mathbb{Z} \times Y, X)$. From Lemma 2.6, it follows that $H(n, x)$ is bounded on $\mathbb{Z} \times \Omega$. On the other hand, as $W(n, x) \in A A P_{0}(\mathbb{Z} \times Y, X)$, then $W(n, x)$ is bounded on $\mathbb{Z} \times Y$. Thus $G(n, x)=H(n, x)+W(n, x)$ is bounded on $\mathbb{Z} \times \Omega$.

In the following, we give the definitions of the discrete $\alpha$-resolvent family, the fractional sum and fractional difference of $\alpha$.

Let $\alpha>0$, defined a sequence $\left\{k_{\alpha}(n)\right\}_{n \in \mathbb{N}}$ by

$$
k_{\alpha}(n):=\frac{\Gamma(n+\alpha)}{\Gamma(\alpha) \Gamma(n+1)},
$$

where $\Gamma$ is the Gamma function. Note that $k_{\alpha}$ satisfies the semigroup property,

$$
\left(k_{\alpha} * k_{\beta}\right)(n)=\sum_{j=0}^{n} k_{\alpha}(n-j) k_{\beta}(j)=k_{\alpha+\beta}(n), \quad \forall n \in \mathbb{N}, \alpha, \beta>0 .
$$

We define the forward Euler operator $\triangle: s(\mathbb{Z}, X) \rightarrow s(\mathbb{Z}, X)$ as

$$
\Delta \gamma(n):=\gamma(n+1)-\gamma(n), \quad n \in \mathbb{Z} .
$$

Recursively, for each $k \in \mathbb{N}$, define $\Delta^{k+1}=\Delta \Delta^{k}=\Delta^{k} \Delta$, and $\Delta^{0}=I$ is the identity operator.

Definition 2.7 (Abadias and Lizama [28]) Assume $\alpha>0$ and $A$ is a closed linear operator with domain $D(A) \subset X$. An operator sequence $\left\{S_{\alpha}(n)\right\}_{n \in \mathbb{N}} \subset \mathbb{L}(X)$ is said to be a discrete $\alpha$-resolvent family generated by $A$ if for any $n \in \mathbb{N}$ and $x \in D(A)$

$$
S_{\alpha}(n) A x=A S_{\alpha}(n) x, \quad S_{\alpha}(n) x=k_{\alpha}(n) x+A\left(k_{\alpha} * S_{\alpha}\right)(n) x .
$$

Definition 2.8 (Abadias and Lizama [28]) For any $\alpha>0$, let $\rho(n)=|n|^{\alpha-1}, n \in \mathbb{Z}$, and $f \in$ $l_{\rho}(\mathbb{Z}, X)$ be a sequence. The fractional sum of $f$ is given by

$$
\triangle^{-\alpha} f(n):=\sum_{j=-\infty}^{n} k_{\alpha}(n-j) f(j), \quad n \in \mathbb{Z}
$$

Definition 2.9 (Abadias and Lizama [28]) For any $\alpha>0$, let $\rho(n)=|n|^{\alpha-1}, n \in \mathbb{Z}$, and $f \in$ $l_{\rho}(\mathbb{Z}, X)$ be a sequence. The fractional difference of $f$ is given by

$$
\triangle^{\alpha} f(n):=\triangle^{p} \triangle^{-(p-\alpha)} f(n), \quad n \in \mathbb{Z},
$$

where $p=[\alpha]+1,[\cdot]$ is the largest integer function.

Finally, we give a compactness criterion, the Leray-Schauder alternative theorem and Matkowski's fixed point theorem.

Assume $h: \mathbb{Z} \rightarrow \mathbb{R}^{+}$is a sequence satisfying

$$
h(n) \geq 1, \quad \forall n \in \mathbb{Z}, \quad h(n) \rightarrow+\infty \quad \text { when }|n| \rightarrow+\infty .
$$


Define

$$
C_{h}^{0}(\mathbb{Z}, X):=\left\{\xi: \mathbb{Z} \rightarrow X: \lim _{|n| \rightarrow+\infty} \frac{\|\xi(n)\|}{h(n)}=0\right\}
$$

It is clear that $C_{h}^{0}(\mathbb{Z}, X)$ is a Banach space under the norm

$$
\|\xi\|_{h}:=\sup _{n \in \mathbb{Z}} \frac{\|\xi(n)\|}{h(n)} .
$$

From Cuevas and Pinto (see [34]), we have the following lemma.

Lemma 2.15 (Agarwal, Cuevas and Dantas [35]) Let $S$ be a subset of $C_{h}^{0}(\mathbb{Z}, X)$. $S$ is relatively compact in $C_{h}^{0}(\mathbb{Z}, X)$ if

(i) for all $n \in \mathbb{Z}$, the set $H_{n}(S):=\left\{\frac{x(n)}{h(n)}: x \in S\right\}$ is relatively compact in $X$,

(ii) $S$ is weighted equiconvergent at $\pm \infty$, i.e., $\forall \varepsilon>0, \exists N>0$ s.t. $\|x(n)\|<\varepsilon h(n)$ for $|n| \geq N$ and $x \in S$.

Lemma 2.16 (Matkowski's fixed point theorem [36]) Let $\Lambda: \Xi \rightarrow \Xi$ be a map which is defined on a complete metric space $(\Xi, d)$ satisfying

$$
d(\Lambda x, \Lambda y) \leq \Psi(d(x, y)) \quad \forall x, y \in \Xi
$$

where $\Psi: \mathbb{R}^{+} \rightarrow \mathbb{R}^{+}$is a nondecreasing function satisfying

$$
\lim _{n \rightarrow \infty} \Psi^{n}(t)=0, \quad \forall t>0
$$

Then there is a unique fixed point $z \in X$ such that $\Lambda z=z$.

Lemma 2.17 (Leray-Schauder alternative theorem [37]) Let $\Lambda: \Omega \rightarrow \Omega$ be a completely continuous map defined on $\Omega$, which is a closed convex subset of $X$ satisfying $0 \in \Omega$. Then $\Lambda$ has a fixed point in $\Omega$ or the collection

$$
\{x \in \Omega: x=\lambda \Lambda(x), 0<\lambda<1\}
$$

is unbounded.

\section{Asymptotically almost periodicity of linear fractional difference equations}

In this section, we formulate and prove conditions for the existence of mild solutions with asymptotically almost periodicity of the nonhomogeneous linear difference equations of fractional order given by

$$
\triangle^{\alpha} x(n)=A x(n+1)+F(n), \quad n \in \mathbb{Z},
$$

where $\alpha \in(0,1)$, the operator $A$ generates a $C_{0}$-semigroup on $X, \Delta^{\alpha}$ denotes the Weyllike fractional difference operator, $F: \mathbb{Z} \rightarrow X$ is a discrete asymptotically almost periodic sequence. 
Lemma 3.1 (Abadias and Lizama [28]) Assume A generates a $C_{0}$-semigroup $\{T(t)\}_{t \geq 0}$ on $X$, which is exponentially stable, i.e.

$$
\|T(t)\|_{\mathbb{L}(X)} \leq M e^{-\delta t} \quad \text { for } \forall t>0 \text { and some constants } M>0, \delta>0 .
$$

Then there exists a discrete $\alpha$-resolvent family $\left\{S_{\alpha}(n)\right\}_{n \in \mathbb{N}}$ generated by $A$, which is given by

$$
S_{\alpha}(n) x:=\int_{0}^{\infty} \int_{0}^{\infty} e^{-t} \frac{t^{n}}{n !} f_{s, \alpha}(t) T(s) x \mathrm{~d} s \mathrm{~d} t, \quad n \in \mathbb{N}, x \in X,
$$

where $f_{s, \alpha}(t)$ is said to be a stable Lévy process, which is given by

$$
f_{s, \alpha}(t)=\frac{1}{2 \pi i} \int_{\sigma-i \infty}^{\sigma+i \infty} e^{z \lambda-t z^{\alpha}} \mathrm{d} z, \quad \sigma>0, s>0, t \geq 0,0<\alpha<1 .
$$

Moreover, $\left\{S_{\alpha}(n)\right\}_{n \in \mathbb{N}}$ is summable and

$$
\left\|S_{\alpha}\right\|_{1}=\sum_{k=0}^{\infty}\left\|S_{\alpha}(n)\right\|_{\mathbb{L}(X)} \leq \frac{1}{\delta \alpha \pi}\left(\frac{\pi}{2}-\arctan (\cot (\alpha \pi))\right)=\frac{1}{\delta} .
$$

From the Gearhart-Prüss-Greiner theorem, Abadias and Lizama obtained the following remarkable result.

Lemma 3.2 (Abadias and Lizama [28]) Let $H$ be a Hilbert space, assume A generate a $C_{0}$-semigroup on $H$ satisfying

$$
\{\eta \in \mathbb{C}: \operatorname{Re}(\eta)>0\} \subset \rho(A), \sup _{\operatorname{Re}(\eta)>0}(\eta-A)^{-1}<\infty .
$$

Then there is a discrete $\alpha$-resolvent family $\left\{S_{\alpha}(n)\right\}_{n \in \mathbb{N}} \subset \mathbb{L}(X)$ generated by $A$, which is summable.

Definition 3.1 (Abadias and Lizama [28]) Assume $\left\{S_{\alpha}(n)\right\}_{n \in \mathbb{N}} \subset \mathbb{L}(X)$ is a discrete $\alpha$ resolvent family generated by $A$. If for each $n \in \mathbb{Z}, m \rightarrow S_{\alpha}(m) F(n-1-m)$ is summable on $\mathbb{N}$. Then the sequence

$$
x(n)=\sum_{k=-\infty}^{n-1} S_{\alpha}(n-1-k) F(k), \quad n \in \mathbb{Z},
$$

is said to be a mild solution for equation (3.1).

Theorem 3.1 Assume $\left\{S_{\alpha}(n)\right\}_{n \in \mathbb{N}} \subset \mathbb{L}(X)$ is a discrete $\alpha$-resolvent family generated by $A$. If $F \in A A P(\mathbb{Z}, X)$, then there exists an asymptotically almost periodic mild solution of Eq. (3.1), which is given by

$$
x(n)=\sum_{k=-\infty}^{n-1} S_{\alpha}(n-1-k) F(k), \quad n \in \mathbb{Z} .
$$


Proof $\left\{S_{\alpha}(n)\right\}_{n \in \mathbb{N}} \subset \mathbb{L}(X)$ is a discrete $\alpha$-resolvent family generated by $A$, which is summable. Then one has

$$
\begin{aligned}
\|x(n)\| & \leq \sum_{k=-\infty}^{n-1}\left\|S_{\alpha}(n-1-k)\right\|\|F(k)\|=\sum_{k=0}^{+\infty}\left\|S_{\alpha}(k)\right\|\|F(n-1-k)\| \\
& \leq\|F\|_{d} \sum_{k=0}^{+\infty}\left\|S_{\alpha}(k)\right\|=\|F\|_{d}\left\|S_{\alpha}\right\|_{1}<+\infty, \quad \forall n \in \mathbb{Z} .
\end{aligned}
$$

Thus

$$
x(n)=\sum_{k=-\infty}^{n-1} S_{\alpha}(n-1-k) F(k), \quad n \in \mathbb{Z},
$$

is a mild solution to Eq. (3.1).

Next we show that $x(n) \in A A P(\mathbb{Z}, X)$.

Indeed, since $F \in A A P(\mathbb{Z}, X)$, we have $F(n)=G(n)+H(n)$ with $G(n) \in A P(\mathbb{Z}, X)$ and $H(n) \in A A P_{0}(\mathbb{Z}, X)$, thus

$$
\begin{aligned}
x(n) & =\sum_{k=-\infty}^{n-1} S_{\alpha}(n-1-k) G(k)+\sum_{k=-\infty}^{n-1} S_{\alpha}(n-1-k) H(k) \\
& =\sum_{k=0}^{+\infty} S_{\alpha}(k) G(n-1-k)+\sum_{k=-\infty}^{n-1} S_{\alpha}(n-1-k) H(k) \\
& :=P(n)+Q(n), \quad n \in \mathbb{Z} .
\end{aligned}
$$

According to Lemma 2.5 , we get $P(n) \in A P(\mathbb{Z}, X)$.

As $H(n) \in A A P_{0}(\mathbb{Z}, X)$, then $\forall \varepsilon>0, \exists N=N(\varepsilon)>0$ s.t.

$$
\|H(k)\| \leq \varepsilon, \quad \forall k>N
$$

Thus

$$
\begin{aligned}
\|Q(n)\| & =\left\|\sum_{k=-\infty}^{n-1} S_{\alpha}(n-1-k) H(k)\right\| \leq \sum_{k=-\infty}^{n-1}\left\|S_{\alpha}(n-1-k)\right\|\|H(k)\| \\
& =\sum_{k=0}^{+\infty}\left\|S_{\alpha}(k)\right\|\|H(n-1-k)\| \leq \varepsilon \sum_{k=0}^{+\infty}\|S(k)\|=\varepsilon\left\|S_{\alpha}\right\|_{1}, \quad \forall n>N+k-1,
\end{aligned}
$$

which implies $Q(n) \in A A P_{0}(\mathbb{Z}, X)$.

Then

$$
x(n)=\sum_{k=-\infty}^{n-1} S_{\alpha}(n-1-k) F(k), \quad n \in \mathbb{Z},
$$

is a mild solution of Eq. (3.1), and it is asymptotically almost periodic.

According to Lemma 3.1, we can get the following corollary. 
Corollary 3.1 Assume the $C_{0}$-semigroup on $X$ generated by $A$ satisfies (3.2). If $F \in$ $A A P(\mathbb{Z}, X)$, then there is an asymptotically almost periodic mild solution of $E q$. (3.1) given by

$$
x(n)=\sum_{k=-\infty}^{n-1} S_{\alpha}(n-1-k) F(k), \quad n \in \mathbb{Z},
$$

where $\left\{S_{\alpha}(n)\right\}_{n \in \mathbb{N}}$ is given by Lemma 3.1.

From Lemma 3.2, we have the following corollary.

Corollary 3.2 Let $H$ be a Hilbert space, assume A generates a $C_{0}$-semigroup on $H$ satisfying

$$
\{\eta \in \mathbb{C}: \operatorname{Re}(\eta)>0\} \subset \rho(A), \quad \sup _{\operatorname{Re}(\eta)>0}(\eta-A)^{-1}<\infty .
$$

Then there is a discrete $\alpha$-resolvent family $\left\{S_{\alpha}(n)\right\}_{n \in \mathbb{N}} \subset \mathbb{L}(H)$ generated by $A$, which is summable (by Lemma 3.2). If $F \in A A P(\mathbb{Z}, H)$, then there is an asymptotically almost periodic mild solution of Eq. (3.1) in H given by

$$
x(n)=\sum_{k=-\infty}^{n-1} S_{\alpha}(n-1-k) F(k), \quad n \in \mathbb{Z} .
$$

\section{Asymptotically almost periodicity of nonlinear fractional difference equations}

In this section, we will state and prove conditions for the existence of mild solutions with asymptotically almost periodicity of the nonhomogeneous nonlinear difference equations of fractional order given by

$$
\triangle^{\alpha} x(n)=A x(n+1)+F(n, x(n)), \quad n \in \mathbb{Z},
$$

where $\alpha \in(0,1)$, the operator $A$ generates a $C_{0}$-semigroup on $X, \Delta^{\alpha}$ denotes the Weyl-like fractional difference operator, $F(n, x): \mathbb{Z} \times X \rightarrow X$ is a function to be specified later.

Definition 4.1 (Abadias and Lizama [28]) Assume $\left\{S_{\alpha}(n)\right\}_{n \in \mathbb{N}} \subset \mathbb{L}(X)$ is a discrete $\alpha$ resolvent family generated by $A$. A sequence $x \in s(\mathbb{Z}, X)$ is said to be a mild solution of Eq. (4.1) if for each $n \in \mathbb{Z}, m \rightarrow S_{\alpha}(m) F(n-1-m, x(n-1-m))$ is summable on $\mathbb{N}$, and $x$ satisfies

$$
x(n)=\sum_{k=-\infty}^{n-1} S_{\alpha}(n-1-k) F(k, x(k)), \quad n \in \mathbb{Z} .
$$

Now we give a composition theorem of discrete asymptotically almost periodic sequences. 
Lemma 4.1 Assume $\{T(n)\}_{n \in \mathbb{N}}$ is a summable sequence. Then, for any discrete asymptotically almost periodic sequence $\phi: \mathbb{Z} \rightarrow X$, the sequence $T * \beta$ given by

$$
(T * \varphi)(n):=\sum_{k=-\infty}^{n-1} T(n-1-k) \varphi(k), \quad n \in \mathbb{Z}
$$

is discrete asymptotically almost periodic.

Proof Note that

$$
\begin{aligned}
\|(T * \varphi)(n)\| & \leq \sum_{k=-\infty}^{n-1}\|T(n-1-k)\|\|\varphi(k)\|=\sum_{k=0}^{+\infty}\|T(k)\|\|\varphi(n-1-k)\| \\
& \leq\|\varphi\|_{d} \sum_{k=0}^{+\infty}\|T(k)\| \leq\|\varphi\|_{d}\|T\|_{1}<+\infty
\end{aligned}
$$

hence $T * \varphi$ is well defined.

Next, we prove that $(T * \varphi)(n) \in A A P(\mathbb{Z}, X)$.

Indeed, since $\varphi \in A A P(\mathbb{Z}, X)$, then $\varphi(n)=\varphi_{1}(n)+\varphi_{2}(n)$ with $\varphi_{1}(n) \in A P(\mathbb{Z}, X), \varphi_{2}(n) \in$ $A A P_{0}(\mathbb{Z}, X)$, thus

$$
\begin{aligned}
(T * \varphi)(n) & =\sum_{k=-\infty}^{n-1} S(n-1-k) \varphi_{1}(k)+\sum_{k=-\infty}^{n-1} T(n-1-k) \varphi_{2}(k) \\
& =\sum_{k=0}^{+\infty} T(k) \varphi_{1}(n-1-k)+\sum_{k=-\infty}^{n-1} T(n-1-k) \varphi_{2}(k) \\
& :=P(n)+Q(n), \quad n \in \mathbb{Z} .
\end{aligned}
$$

According to Lemma 2.5 , we get $P(n) \in A P(\mathbb{Z}, X)$.

As $\varphi_{2}(n) \in A A P_{0}(\mathbb{Z}, X)$, then $\forall \varepsilon>0, \exists N=N(\varepsilon)>0$ s.t.

$$
\left\|\varphi_{2}(k)\right\| \leq \varepsilon, \quad \forall k>N
$$

Thus

$$
\begin{aligned}
\|Q(n)\| & =\left\|\sum_{k=-\infty}^{n-1} T(n-1-k) \varphi_{2}(k)\right\| \leq \sum_{k=-\infty}^{n-1}\|T(n-1-k)\|\left\|\varphi_{2}(k)\right\| \\
& =\sum_{k=0}^{+\infty}\|T(k)\|\left\|\varphi_{2}(n-1-k)\right\| \leq \varepsilon \sum_{k=0}^{+\infty}\|T(k)\| \leq \varepsilon\|T\|_{1}, \quad \forall n>N+k,
\end{aligned}
$$

which implies $Q(n) \in A A P_{0}(\mathbb{Z}, X)$.

Lemma 4.2 Let $F \in U C(\mathbb{Z} \times X, X) \cap A A P(\mathbb{Z} \times X, X)$ and $\Omega$ be any compact set in $X$. Assume $x \in A P(\mathbb{Z}, X)$ and $x(n) \in \Omega$. Then $U(n):=F(n, x(n))$ is a discrete asymptotically almost periodic sequence. 
Proof Let

$$
F(n, x)=G(n, x)+H(n, x), \quad x(n)=\varphi(n)+\psi(n),
$$

with

$$
\begin{aligned}
& G(n, x) \in A P(\mathbb{Z} \times X, X), \quad H(n, x) \in A A P_{0}(\mathbb{Z} \times X, X), \\
& \varphi(n) \in A P(\mathbb{Z}, X), \quad \psi(n) \in A A P_{0}(\mathbb{Z}, X) .
\end{aligned}
$$

As $x(n) \in \Omega$, then $\varphi(n) \in \Omega$ (by Lemma 2.13). And Lemma 2.9 guarantees that $G(\cdot, \varphi(\cdot)) \in$ $A P(\mathbb{Z}, X)$. Consequently, it suffices to prove the sequence $\beta$ given by

$$
\beta(\cdot):=F(\cdot, x(\cdot))-G(\cdot, \varphi(\cdot))=(F(\cdot, x(\cdot))-F(\cdot, \varphi(\cdot)))+H(\cdot, \varphi(\cdot))
$$

belongs to $A A P_{0}(\mathbb{Z}, X)$.

As $F \in U C(\mathbb{Z} \times X, X)$, one has $\forall \varepsilon>0, \exists \delta>0$ s.t.

$$
\|F(n, x)-F(n, y)\| \leq \frac{\varepsilon}{2}, \quad \forall n \in \mathbb{Z} x, y \in X \text { with }\|x-y\| \leq \delta .
$$

For the above $\varepsilon$ and $\delta$, as $H(n, x) \in A A P_{0}(\mathbb{Z} \times X, X)$ and $\psi(n) \in A A P_{0}(\mathbb{Z}, X)$, together with $\varphi(\mathbb{Z}) \subset \overline{x(\mathbb{Z})}$ which follows from Lemma 2.13, we have $\exists N=N(\delta)>0$ s.t.

$$
\begin{aligned}
& \|H(n, \varphi(n))\| \leq \frac{\varepsilon}{2}, \quad \forall n>N \text { uniformly for } \varphi(n) \in \Omega, \\
& \|x(n)-\varphi(n)\|=\|\psi(n)\| \leq \delta, \quad \forall n>N .
\end{aligned}
$$

Thus

$$
\|\beta(n)\|=\|F(n, x(n))-F(n, \varphi(n))\|+\|H(n, \varphi(n))\| \leq \varepsilon, \quad \forall n>N,
$$

which implies $\beta(\cdot) \in A A P_{0}(\mathbb{Z}, X)$.

Corollary 4.1 Assume $F \in A A P(\mathbb{Z} \times X, X)$ and satisfies a Lipschitz condition in $x \in X$ uniformly for $n \in \mathbb{Z}$, i.e.

$$
\|F(n, x)-F(n, y)\| \leq L\|x-y\| \quad \text { for } \forall x, y \in X, n \in \mathbb{Z} \text { and some constant } L .
$$

Then the conclusion of Lemma 4.2 also holds.

Corollary 4.2 The conclusion of Lemma 4.2 holds if $F \in A A P(\mathbb{Z} \times X, X)$ and satisfies

$$
\|F(n, x)-F(n, y)\| \leq \Phi(\|x-y\|), \quad \forall n \in \mathbb{Z}, x, y \in X,
$$

with $\Phi: \mathbb{R}^{+} \rightarrow \mathbb{R}^{+}$being a linear nondecreasing function.

Lemma 4.3 Let $F \in U C_{n}(\mathbb{Z} \times X, X) \cap A A P(\mathbb{Z} \times X, X)$ and $\Omega$ be any compact set in $X$. Assume $x \in A P(\mathbb{Z}, X)$ and $x(n) \in \Omega$. Then $U(n)=F(n, x(n))$ is discrete asymptotically almost periodic. 
Proof Let

$$
F(n, x)=G(n, x)+H(n, x), \quad x(n)=\varphi(n)+\psi(n),
$$

with

$$
\begin{aligned}
& G(n, x) \in A P(\mathbb{Z} \times X, X), \quad G(n, x) \in A A P_{0}(\mathbb{Z} \times X, X), \\
& \varphi(n) \in A P(\mathbb{Z}, X), \quad \psi(n) \in A A P_{0}(\mathbb{Z}, X) .
\end{aligned}
$$

Similar to the proof of Lemma 4.2, it suffices to prove the sequence $\beta$ defined by

$$
\beta(\cdot):=F(\cdot, x(\cdot))-G(\cdot, \varphi(\cdot))=(F(\cdot, x(\cdot))-F(\cdot, \varphi(\cdot)))+H(\cdot, \varphi(\cdot))
$$

belongs to $A A P_{0}(\mathbb{Z}, X)$.

As $F \in U C_{n}(\mathbb{Z} \times X, X)$, then $\forall \varepsilon>0, \exists \delta>0$ s.t.

$$
\|F(n, x)-F(n, y)\| \leq L_{F}(n) \varepsilon, \quad \forall n \in \mathbb{Z} x, y \in X \text { with }\|x-y\| \leq \delta .
$$

For the above $\varepsilon$ and $\delta$, as $h(n, x) \in A A P_{0}(\mathbb{Z} \times X, X)$ and $\psi(n) \in A A P_{0}(\mathbb{Z}, X)$, together with $p(\mathbb{Z}) \subset \overline{x(\mathbb{Z})}$ which follows from Lemma 2.13, then $\exists N=N(\delta)>0$ s.t.

$$
\begin{aligned}
& \|H(n, \varphi(n))\| \leq \varepsilon, \quad \forall n>N \text { uniformly for } \varphi(n) \in \Omega, \\
& \|x(n)-\varphi(n)\|=\|\psi(n)\| \leq \delta, \quad \forall n>N .
\end{aligned}
$$

Thus, for $n>N$, together with $L_{F} \in L(\mathbb{Z})$, we have

$$
\begin{aligned}
\|\beta(n)\| & =\|F(n, x(n))-F(n, \varphi(n))\|+\|H(n, \varphi(n))\| \\
& \leq L_{F}(n) \varepsilon+\varepsilon \leq\left(\left\|L_{F}\right\|_{l}+1\right) \varepsilon,
\end{aligned}
$$

which implies $\beta(\cdot) \in A A P_{0}(\mathbb{Z}, X)$.

Corollary 4.3 Assume $F \in A A P(\mathbb{Z} \times X, X)$ and satisfies a local Lipschitz condition in $x \in X$ uniformly for $n \in \mathbb{Z}$, i.e. for each $r>0$, and $\forall x, y \in X$ with $\|x\| \leq r,\|y\| \leq r$,

$$
\|F(n, x)-F(n, y)\| \leq L_{F}(r)\|x-y\|, \quad \forall n \in \mathbb{Z},
$$

where $L_{F}: \mathbb{R}^{+} \rightarrow \mathbb{R}^{+}$is a function. Then the conclusion of Lemma 4.3 also holds.

Now we are in a position to show the asymptotic almost periodicity of mild solutions to Eq. (4.1). To prove the results, let us introduce the following assumptions:

$\left(\mathrm{H}_{0}\right)$ For all $n_{1}, n_{2} \in \mathbb{Z}, n_{1} \leq n_{2}$ and $\eta>0$, the collection $\left\{F(n, x): n_{1} \leq n \leq n_{2},\|x\| \leq \eta\right\}$ is relatively compact in $X$.

$\left(\mathrm{H}_{1}\right)$ The discrete $\alpha$-resolvent family $\left\{S_{\alpha}(n)\right\}_{n \in \mathbb{N}} \subset \mathbb{L}(X)$ generated by $A$ is summable.

$\left(\mathrm{H}_{2}\right) F(n, x) \in A A P(\mathbb{Z} \times X, X)$ and it is bounded on $\mathbb{Z} \times X$.

Firstly, we give an important lemma. 
Lemma 4.4 Assume $\left(\mathrm{H}_{0}\right),\left(\mathrm{H}_{1}\right)$ and $\left(\mathrm{H}_{2}\right)$ hold. Let $x(n)$ be given by (4.2), then the collection $\overline{\{x(n): n \in \mathbb{Z}\}}$ is compact in $X$.

Proof From $\left(\mathrm{H}_{2}\right)$, it follows that $\exists M>0$ s.t. $\|F(n, x)\|<M$ for $\forall n \in \mathbb{Z}$ and $x \in X$. This, together with $\left(\mathrm{H}_{1}\right)$, implies that

$$
\begin{aligned}
\|x(n)\| & \leq \sum_{i=-\infty}^{n-1}\left\|S_{\alpha}(n-1-i)\right\|\|F(i, x(i))\|=\sum_{i=0}^{+\infty}\left\|S_{\alpha}(i)\right\|\|F(n-1-i, x(n-1-i))\| \\
& \leq M \sum_{i=0}^{+\infty}\left\|S_{\alpha}(i)\right\|=M\left\|S_{\alpha}\right\|_{1}<+\infty \quad \text { for all } n \in \mathbb{Z} .
\end{aligned}
$$

From $\left(\mathrm{H}_{1}\right)$, it follows that, for $\varepsilon>0$, one can choose $m \in \mathbb{Z}^{+}$s.t.

$$
M \sum_{i=m}^{\infty}\left\|S_{\alpha}(i)\right\| \leq \varepsilon
$$

Since

$$
\begin{aligned}
x(n) & =\sum_{i=-\infty}^{n-1} S_{\alpha}(n-1-i) F(i, x(i)) \\
& =\sum_{i=0}^{+\infty} S_{\alpha}(i) F(n-1-i, x(n-1-i)) \\
& =\sum_{i=0}^{m-1} S_{\alpha}(i) F(n-1-i, x(n-1-i))+\sum_{i=m}^{\infty} S_{\alpha}(i) F(n-1-i, x(n-1-i)),
\end{aligned}
$$

noting that

$$
\begin{aligned}
\left\|\sum_{i=m}^{\infty} S_{\alpha}(i) F(n-1-i, x(n-1-i))\right\| & \leq \sum_{i=m}^{\infty}\left\|S_{\alpha}(i)\right\|\|F(n-1-i, x(n-1-i))\| \\
& \leq M \sum_{i=m}^{\infty}\left\|S_{\alpha}(i)\right\| \leq \varepsilon,
\end{aligned}
$$

one has

$$
x(n) \in \overline{\operatorname{co}(S)}+B_{\varepsilon}(X)
$$

here $\overline{\operatorname{co}(S)}$ is the convex hull of $S$ and

$$
S=\bigcup_{i=0}^{m-1}\left\{S_{\alpha}(i) F(\tau, x): \tau \in[n-l, n-1] \cap \mathbb{Z},\|x\| \leq M\left\|S_{\alpha}\right\|_{1}\right\} .
$$

It follows from $\left(\mathrm{H}_{0}\right)$ that $S$ is relatively compact in $X$, this together with

$$
\{x(n): n \in \mathbb{Z}\} \subseteq \overline{\operatorname{co}(S)}+B_{\varepsilon}(X),
$$


shows that the collection $\{x(n): n \in \mathbb{Z}\}$ is relatively compact in $X$. Then $\overline{\{x(n): n \in \mathbb{Z}\}}$ is compact in $X$.

Remark 4.1 In Lemma 2.14, we prove that a discrete asymptotically almost periodic function $F(n, x): \mathbb{Z} \times X \rightarrow X$ is bounded only on $\mathbb{Z} \times \Omega$ for any compact set $\Omega$ in $X$, so the condition boundedness in $\left(\mathrm{H}_{2}\right)$ does not conflict with the condition $F(n, x) \in A A P(\mathbb{Z} \times X, X)$.

Firstly, we state and prove the existence and uniqueness of mild solutions with asymptotically almost periodicity of Eq. (4.1) when the perturbation $F$ is Lipschitz type.

$\left(\mathrm{H}_{3}\right) F$ satisfies the following Lipschitz condition:

$$
\|F(n, x)-F(n, y)\| \leq L_{F}\|x-y\|, \quad \forall n \in \mathbb{Z}, x, y \in X,
$$

where $L_{F}>0$ is a constant.

Theorem 4.1 Assume $\left(\mathrm{H}_{0}\right),\left(\mathrm{H}_{1}\right),\left(\mathrm{H}_{2}\right)$ and $\left(\mathrm{H}_{3}\right)$ hold. Then there is a unique mild solution of Eq. (4.1) whenever $L_{F}\left\|S_{\alpha}\right\|_{1}<1$. Moreover, the mild solution is asymptotically almost periodic.

Proof Let $\mathbb{F}: A A P(\mathbb{Z}, X) \rightarrow A A P(\mathbb{Z}, X)$ be the map given by

$$
(\mathbb{F} x)(n):=\sum_{i=-\infty}^{n-1} S_{\alpha}(n-1-i) F(i, x(i)) .
$$

Since $\left\{S_{\alpha}(n)\right\}_{n \in \mathbb{N}} \subset \mathbb{L}(X)$ is a discrete $\alpha$-resolvent family generated by $A$, and it is summable, we have

$$
\begin{aligned}
\|(\mathbb{F} x)(n)\| & \leq \sum_{i=-\infty}^{n-1}\left\|S_{\alpha}(n-1-i)\right\|\|F(i, x(i))\|=\sum_{i=0}^{+\infty}\left\|S_{\alpha}(i)\right\|\|F(n-1-i, x(n-1-i))\| \\
& \leq\|F\| \sum_{i=0}^{+\infty}\left\|S_{\alpha}(i)\right\|=\|F\|\left\|S_{\alpha}\right\|_{1}<+\infty \quad \text { for all } n \in \mathbb{Z} .
\end{aligned}
$$

From Corollary 4.1, together with $x \in A A P(\mathbb{Z}, X), F(n, x)$ satisfying $\left(\mathrm{H}_{3}\right)$ and Lemma 4.4, it follows that $F(\cdot, x(\cdot))$ is in $A A P(\mathbb{Z}, X)$. Furthermore it follows from Lemma 4.1 that $\mathbb{F} x$ is in $A A P(\mathbb{Z}, X)$. Hence $\mathbb{F}$ is well defined.

In addition, for $x, y \in A A P(\mathbb{Z}, X)$, one has

$$
\begin{aligned}
\|(\mathbb{F} x)(n)-(\mathbb{F} y)(n)\| & =\left\|\sum_{i=-\infty}^{n-1} S_{\alpha}(n-1-i)[F(i, x(i))-F(i, y(i))]\right\| \\
& \leq \sum_{i=-\infty}^{n-1}\left\|S_{\alpha}(n-1-i)\right\|\|F(i, x(i))-F(i, y(i))\| \\
& \leq L_{F} \sum_{i=-\infty}^{n-1}\left\|S_{\alpha}(n-1-i)\right\|\|x(i)-y(i)\| \\
& \leq\left\|S_{\alpha}\right\|_{1} L_{F}\|x-y\|_{d},
\end{aligned}
$$


which implies

$$
\|(\mathbb{F} x)(n)-(\mathbb{F} y)(n)\|_{d} \leq\left\|S_{\alpha}\right\|_{1} L_{F}\|x-y\|_{d} .
$$

Recalling that $\left\|S_{\alpha}\right\|_{1} L_{F}<1$, then $\mathbb{F}$ is a contraction on $A A P(\mathbb{Z}, X)$. and thus, there is a unique sequence $x \in A A P(\mathbb{Z}, X)$ satisfying

$$
x(n)=\sum_{i=-\infty}^{n-1} S_{\alpha}(n-1-i) F(i, x(i)), \quad n \in \mathbb{Z},
$$

which shows that $x$ is a unique mild solution of Eq. (4.1) and it is discrete asymptotically almost periodic.

Following Lemma 3.2, we have the following corollary.

Corollary 4.4 Let $H$ be a Hilbert space, assume A generates a $C_{0}$-semigroup on $H$ satisfying

$$
\{\eta \in \mathbb{C}: \operatorname{Re}(\eta)>0\} \subset \rho(A), \quad \sup _{\operatorname{Re}(\eta)>0}(\eta-A)^{-1}<\infty .
$$

Then there is a discrete $\alpha$-resolvent family $\left\{S_{\alpha}(n)\right\}_{n \in \mathbb{N}} \subset \mathbb{L}(H)$ generated by $A$, which is summable (by Lemma 3.2). Assume $\left(\mathrm{H}_{0}\right),\left(\mathrm{H}_{2}\right)$ and $\left(\mathrm{H}_{3}\right)$ hold with $X$ instead of $H$. Then there is a unique mild solution of Eq. (4.1) whenever $L_{F}\left\|S_{\alpha}\right\|_{1}<1$. Moreover, the mild solution is asymptotically almost periodic.

According to Lemma 3.1, we can get the following corollary.

Corollary 4.5 Assume the $C_{0}$-semigroup on $X$ generated by A satisfies (3.2), and $\left(\mathrm{H}_{0}\right),\left(\mathrm{H}_{2}\right)$, $\left(\mathrm{H}_{3}\right)$ hold. Then there is a unique mild solution of Eq. (4.1) whenever $L_{F}<\delta$, where $\delta$ is given by Lemma 3.1. Moreover, the mild solution is asymptotically almost periodic.

Theorem 4.1 can be extended to the case of $F$ being locally Lipschitz continuous.

$\left(\mathrm{H}_{4}\right) F(n, x)$ satisfies a local Lipschitz condition in $x \in X$ uniformly for $n \in \mathbb{Z}$, i.e. for each $r>0$, and $\forall x, y \in X$ with $\|x\| \leq r,\|y\| \leq r$,

$$
\|F(n, x)-F(n, y)\| \leq L_{F}(r)\|x-y\|, \quad \forall n \in \mathbb{Z},
$$

where $L_{F}: \mathbb{R}^{+} \rightarrow \mathbb{R}^{+}$is a nondecreasing function.

Theorem 4.2 Assume that $\left(\mathrm{H}_{0}\right),\left(\mathrm{H}_{1}\right),\left(\mathrm{H}_{2}\right),\left(\mathrm{H}_{4}\right)$ hold and if there exists $r_{0}>0$ such that

$$
\left\|S_{\alpha}\right\|_{1}\left(L_{F}\left(r_{0}\right)+\frac{1}{r_{0}} \sup _{i \in \mathbb{Z}}\|F(i, 0)\|\right)<1
$$

Then there is a unique mild solution $x(n)$ of Eq. (4.1), moreover, $x(n)$ is asymptotically almost periodic and $\|x(n)\| \leq r_{0}$. 
Proof Let

$$
\mathbb{F}: B_{r_{0}}(A A P(\mathbb{Z}, X)) \rightarrow B_{r_{0}}(A A P(\mathbb{Z}, X))
$$

be the map defined by (4.3). Since $x \in A A P(\mathbb{Z}, X)$ and $F(n, x)$ satisfies $\left(\mathrm{H}_{4}\right)$, together with Lemma 4.4, one can see by Corollary 4.3 that $F(\cdot, x(\cdot))$ is in $A A P(\mathbb{Z}, X)$. Furthermore it follows from Lemma 4.1 that $\mathbb{F} x$ is in $A A P(\mathbb{Z}, X)$. Let $x$ be in $B_{r_{0}}(A A P(\mathbb{Z}, X))$, we have the following estimates:

$$
\begin{aligned}
\|(\mathbb{F} x)(n)\| & =\left\|\sum_{i=-\infty}^{n-1} S_{\alpha}(n-1-i) F(i, x(i))\right\| \\
& \leq \sum_{i=-\infty}^{n-1}\left\|S_{\alpha}(n-1-i)\right\|\|F(i, x(i))-F(i, 0)\|+\sum_{i=-\infty}^{n-1}\left\|S_{\alpha}(n-1-i)\right\|\|F(i, 0)\| \\
& \leq L_{F}\left(r_{0}\right) \sum_{i=-\infty}^{n-1}\left\|S_{\alpha}(n-1-i)\right\|\|x(i)\|+\left\|S_{\alpha}\right\|_{1} \sup _{i \in \mathbb{Z}}\|F(i, 0)\| \\
& \leq\left\|S_{\alpha}\right\|_{1}\left(L_{F}\left(r_{0}\right)+\frac{\sup _{i \in \mathbb{Z}}\|F(i, 0)\|}{r_{0}}\right) r_{0}<r_{0} .
\end{aligned}
$$

Hence $\mathbb{F} x \in B_{r_{0}}(A A P(\mathbb{Z}, X))$, which implies $\mathbb{F}$ is well defined.

In addition, for $x, y \in B_{r_{0}}(A A P(\mathbb{Z}, X))$, one has

$$
\begin{aligned}
\|(\mathbb{F} x)(n)-(\mathbb{F} y)(n)\| & =\left\|\sum_{i=-\infty}^{n-1} S_{\alpha}(n-1-i)[F(i, x(i))-F(i, y(i))]\right\| \\
& \leq \sum_{i=-\infty}^{n-1}\left\|S_{\alpha}(n-1-i)\right\|\|F(i, x(i))-F(i, y(i))\| \\
& \leq L_{F}\left(r_{0}\right) \sum_{i=-\infty}^{n-1}\left\|S_{\alpha}(n-1-i)\right\|\|x(i)-y(i)\| \\
& \leq\left\|S_{\alpha}\right\|_{1} L_{F}\left(r_{0}\right)\|x-y\|_{d},
\end{aligned}
$$

which implies

$$
\|(\mathbb{F} x)(n)-(\mathbb{F} y)(n)\|_{d} \leq\left\|S_{\alpha}\right\|_{1} L_{F}\left(r_{0}\right)\|x-y\|_{d} .
$$

Recalling that $\left\|S_{\alpha}\right\|_{1} L_{F}\left(r_{0}\right)<1$, then $\mathbb{F}$ is a contraction on $B_{r_{0}}(A A P(\mathbb{Z}, X))$, and thus, there is a unique sequence $x(n) \in B_{r_{0}}(A A P(\mathbb{Z}, X))$ satisfying

$$
x(n)=\sum_{i=-\infty}^{n-1} S_{\alpha}(n-1-i) F(i, x(i)), \quad n \in \mathbb{Z},
$$

which shows $x$ is a unique mild solution of Eq. (4.1), and it is discrete asymptotically almost periodic satisfying $\|x(n)\| \leq r_{0}$.

Following Lemma 3.2, we have the following corollary. 
Corollary 4.6 Let $H$ be a Hilbert space, assume A generates a $C_{0}$-semigroup on $H$ satisfying

$$
\{\eta \in \mathbb{C}: \operatorname{Re}(\eta)>0\} \subset \rho(A), \quad \sup _{\operatorname{Re}(\eta)>0}(\eta-A)^{-1}<\infty .
$$

Then there is a discrete $\alpha$-resolvent family $\left\{S_{\alpha}(n)\right\}_{n \in \mathbb{N}} \subset \mathbb{L}(H)$ generated by $A$, which is summable (by Lemma 3.2). Assume $\left(\mathrm{H}_{0}\right),\left(\mathrm{H}_{2}\right),\left(\mathrm{H}_{4}\right)$ hold with $X$ instead of $H$, and there exists $r_{0}>0$ such that

$$
\left\|S_{\alpha}\right\|_{1}\left(L_{F}\left(r_{0}\right)+\frac{1}{r_{0}} \sup _{i \in \mathbb{Z}}\|F(i, 0)\|\right)<1 .
$$

Then there is a unique mild solution of Eq. (4.1) in H which is asymptotically almost periodic and $\|x(n)\| \leq r_{0}$.

According to Lemma 3.1, we can get the following corollary.

Corollary 4.7 Assume the $C_{0}$-semigroup on $X$ generated by A satisfies (3.2), and $\left(\mathrm{H}_{0}\right),\left(\mathrm{H}_{2}\right)$, $\left(\mathrm{H}_{4}\right)$ hold. If there exists $r_{0}>0$ such that

$$
L_{F}\left(r_{0}\right)+\frac{1}{r_{0}} \sup _{i \in \mathbb{Z}}\|F(i, 0)\|<\delta,
$$

where $\delta$ is given by Lemma 3.1, then there is a unique mild solution of Eq. (4.1) which is asymptotically almost periodic and $\|x(n)\| \leq r_{0}$.

Furthermore, we can obtain the following result.

$\left(\mathrm{H}_{5}\right)$ Assume $F$ satisfies

$$
\|F(n, x)-F(n, y)\| \leq \Phi(\|x-y\|), \quad \forall n \in \mathbb{Z}, x, y \in X,
$$

where $\Phi: \mathbb{R}^{+} \rightarrow \mathbb{R}^{+}$is a linear nondecreasing function.

Theorem 4.3 Assume $\left(\mathrm{H}_{0}\right),\left(\mathrm{H}_{1}\right),\left(\mathrm{H}_{2}\right)$ and $\left(\mathrm{H}_{5}\right)$ hold. Then there is a unique mild solution of Eq. (4.1) which is asymptotically almost periodic if, for each $t>0,\left(\left\|S_{\alpha}\right\|_{1} \Phi\right)^{n}(t) \rightarrow 0$ as $n \rightarrow+\infty$.

Proof Let $\mathbb{F}: A A P(\mathbb{Z}, X) \rightarrow A A P(\mathbb{Z}, X)$ be the map defined by (4.3). Since $A$ generates a discrete $\alpha$-resolvent family $\left\{S_{\alpha}(n)\right\}_{n \in \mathbb{N}} \subset \mathbb{L}(X)$, which is summable, one has

$$
\begin{aligned}
\|(\mathbb{F} x)(n)\| & \leq \sum_{i=-\infty}^{n-1}\left\|S_{\alpha}(n-1-i)\right\|\|F(i, x(i))\|=\sum_{i=0}^{+\infty}\left\|S_{\alpha}(i)\right\|\|F(n-1-i, x(n-1-i))\| \\
& \leq\|F\| \sum_{i=0}^{+\infty}\left\|S_{\alpha}(i)\right\|=\|F\|\left\|S_{\alpha}\right\|_{1}<+\infty \quad \text { for all } n \in \mathbb{Z} .
\end{aligned}
$$

Since $x \in A A P(\mathbb{Z}, X)$ and $F(n, x)$ satisfies $\left(\mathrm{H}_{5}\right)$, together with Lemma 4.4, one can see by Corollary 4.2 that $F(\cdot, x(\cdot))$ is in $A A P(\mathbb{Z}, X)$. Furthermore it follows from Lemma 4.1 that $\mathbb{F} x$ is in $A A P(\mathbb{Z}, X)$. Hence $\mathbb{F}$ is well defined. 
On the other hand, for $x, y \in A A P(\mathbb{Z}, X)$, we have

$$
\begin{aligned}
\|(\mathbb{F} x)(n)-(\mathbb{F} y)(n)\| & =\left\|\sum_{i=-\infty}^{n-1} S_{\alpha}(n-1-i)[F(i, x(i))-F(i, y(i))]\right\| \\
& \leq \sum_{i=-\infty}^{n-1}\left\|S_{\alpha}(n-1-i)\right\|\|F(i, x(i))-F(i, y(i))\| \\
& \leq \sum_{i=-\infty}^{n-1}\left\|S_{\alpha}(n-1-i)\right\| \Phi(\|x(i)-y(i)\|) \\
& \leq\left\|S_{\alpha}\right\|_{1} \Phi\left(\|x-y\|_{d}\right),
\end{aligned}
$$

which implies

$$
\|(\mathbb{F} x)(n)-(\mathbb{F} y)(n)\|_{d} \leq\left(\left\|S_{\alpha}\right\|_{1} \Phi\right)\left(\|x-y\|_{d}\right)
$$

From the Matkowski fixed point theorem (Lemma 2.16), together with the assumption $\left(\left\|S_{\alpha}\right\|_{1} \Phi\right)^{n}(t) \rightarrow 0$ as $n \rightarrow+\infty$ for each $t>0$, it follows that $\mathbb{F}$ has a unique fixed point $x(n) \in A A P(\mathbb{Z}, X)$ such that

$$
x(n)=\sum_{i=-\infty}^{n-1} S_{\alpha}(n-1-i) F(i, x(i)), \quad n \in \mathbb{Z},
$$

which implies that $x$ is a unique mild solution to Eq. (4.1), and it is discrete asymptotically almost periodic.

Following Lemma 3.2, we have the following corollary.

Corollary 4.8 Let $H$ be a Hilbert space, assume A generates a $C_{0}$-semigroup on $H$ satisfying

$$
\{\eta \in \mathbb{C}: \operatorname{Re}(\eta)>0\} \subset \rho(A), \quad \sup _{\operatorname{Re}(\eta)>0}(\eta-A)^{-1}<\infty .
$$

Then there is a discrete $\alpha$-resolvent family $\left\{S_{\alpha}(n)\right\}_{n \in \mathbb{N}} \subset \mathbb{L}(H)$ generated by $A$, which is summable (by Lemma 3.2). Assume $\left(\mathrm{H}_{0}\right),\left(\mathrm{H}_{2}\right),\left(\mathrm{H}_{5}\right)$ hold with $X$ instead of $H$. If for each $t>0,\left(\left\|S_{\alpha}\right\|_{1} \Phi\right)^{n}(t) \rightarrow 0$ as $n \rightarrow+\infty$, then there is a unique mild solution of Eq. (4.1) in $H$ which is asymptotically almost periodic.

According to Lemma 3.1, we can get the following corollary.

Corollary 4.9 Assume the $C_{0}$-semigroup on $X$ generated by A satisfies (3.2), and $\left(\mathrm{H}_{0}\right)$, $\left(\mathrm{H}_{2}\right),\left(\mathrm{H}_{5}\right)$ hold. If for each $t>0,\left(\left\|S_{\alpha}\right\|_{1} \Phi\right)^{n}(t) \rightarrow 0$ as $n \rightarrow+\infty$, then there is a unique mild solution of Eq. (4.1) which is asymptotically almost periodic.

Then we state and prove the existence and uniqueness of mild solutions with asymptotically almost periodicity of Eq. (4.1) when the perturbation $F$ is of non-Lipschitz type, i.e. $F \in U C_{n}(\mathbb{Z} \times X, X)$ or $F \in U C(\mathbb{Z} \times X, X)$. 
Theorem 4.4 Let $h$ be given by Lemma 2.15, and $\left(\mathrm{H}_{0}\right),\left(\mathrm{H}_{1}\right),\left(\mathrm{H}_{2}\right)$ hold, $F \in U C_{n}(\mathbb{Z} \times X, X)$ satisfying the following.

(I) There exist a function $M: \mathbb{Z} \rightarrow \mathbb{R}^{+}$and a nondecreasing function $W: \mathbb{R}^{+} \rightarrow \mathbb{R}^{+}$s.t.

$$
\|F(n, x)\| \leq M(n) W(\|x\|), \quad \forall n \in \mathbb{Z}, x \in X .
$$

(II) For every constant $v>0$,

$$
\lim _{|n| \rightarrow+\infty} \frac{1}{h(n)} \sum_{i=-\infty}^{n-1}\left\|S_{\alpha}(n-1-i)\right\| M(i) W(v h(i))=0 .
$$

(III) For $\forall \varepsilon>0, \exists \delta>0$ s.t.for $\forall x, y \in C_{h}^{0}(\mathbb{Z}, X)$ with $\|x-y\|_{h} \leq \delta$ implies that

$$
\sum_{i=-\infty}^{n-1}\left\|S_{\alpha}(n-1-i)\right\|\|F(i, x(i))-F(i, y(i))\| \leq \varepsilon \quad \text { for all } n \in \mathbb{Z}
$$

(IV) $\liminf _{r \rightarrow+\infty} \frac{r}{\sup _{n \in \mathbb{Z}}\left(\frac{1}{h(n)} \sum_{i=-\infty}^{n-1}\left\|S_{\alpha}(n-1-i)\right\| \| M(i) W(r h(i))\right)}>1$.

Then there is a mild solution of Eq. (4.1) which is asymptotically almost periodic.

The proof of Theorem 4.4 is based on two basic ingredients:

(i) Lemma 2.15 (Cuevas and Pinto [34]), a criterion to obtain the compactness,

(ii) Lemma 2.17 (Granas and Dugundji [37]), the Leray-Schauder alternative theorem to obtain a fixed point.

Proof Define a map $\mathbb{G}: C_{h}^{0}(\mathbb{Z}, X) \rightarrow C_{h}^{0}(\mathbb{Z}, X)$ by

$$
(\mathbb{G} x)(n):=\sum_{i=-\infty}^{n-1} S_{\alpha}(n-1-i) F(i, x(i)), \quad n \in \mathbb{Z} .
$$

We will show that there is a fixed point of $\mathbb{G}$ in $A A P(\mathbb{Z}, X)$. In order to do this by the Leray-Schauder alternative theorem, we show that the conditions in Lemma 2.17 are satisfied. We divide our reasoning into the following seven steps to complete the proof.

Step 1 . The operator $\mathbb{G}$ is well defined.

Let $x$ be in $C_{h}^{0}(\mathbb{Z}, X)$, by condition (I) we have

$$
\begin{aligned}
\|(\mathbb{G} x)(n)\| & =\left\|\sum_{i=-\infty}^{n-1} S_{\alpha}(n-1-i) F(i, x(i))\right\| \\
& \leq \sum_{i=-\infty}^{n-1}\left\|S_{\alpha}(n-1-i)\right\| M(i) W(\|x(n)\|) \\
& \leq \sum_{i=-\infty}^{n-1}\left\|S_{\alpha}(n-1-i)\right\| M(i) W\left(\|x\|_{h} h(i)\right),
\end{aligned}
$$


which implies

$$
\frac{\|(\mathbb{G} x)(n)\|}{h(n)} \leq \frac{1}{h(n)} \sum_{i=-\infty}^{n-1}\left\|S_{\alpha}(n-1-i)\right\| M(i) W\left(\|x\|_{h} h(i)\right) .
$$

From condition (II), it follows that $\mathbb{G}$ is $C_{h}^{0}(\mathbb{Z}, X)$-valued, thus $\mathbb{G}$ is well defined.

Step 2 . The map $\mathbb{G}: C_{h}^{0}(\mathbb{Z}, X) \rightarrow C_{h}^{0}(\mathbb{Z}, X)$ is continuous.

Indeed, for $\forall \varepsilon>0$ and $x, y \in C_{h}^{0}(\mathbb{Z}, X)$ with $\|x-y\|_{h} \leq \delta$, where $\delta>0$ is given by condition (III), from condition (III), it follows that

$$
\begin{aligned}
\|(\mathbb{G} x)(n)-(\mathbb{G} y)(n)\| & =\left\|\sum_{i=-\infty}^{n-1} S_{\alpha}(n-1-i)[F(i, x(i))-F(i, y(i))]\right\| \\
& \leq \sum_{i=-\infty}^{n-1}\left\|S_{\alpha}(n-1-i)\right\|\|F(i, x(i))-F(i, y(i))\| \leq \varepsilon .
\end{aligned}
$$

Note that $h(n) \geq 1$ for every $n \in \mathbb{Z}$, then one can get

$$
\frac{\|(\mathbb{G} x)(n)-(\mathbb{G} y)(n)\|}{h(n)} \leq \varepsilon \quad \forall n \in \mathbb{Z}
$$

this shows that

$$
\|(\mathbb{G} x)(n)-(\mathbb{G} y)(n)\|_{h} \leq \varepsilon \quad \text { for all } n \in \mathbb{Z} .
$$

Since $\varepsilon>0$ is arbitrary, this implies that $\mathbb{G}: C_{h}^{0}(\mathbb{Z}, X) \rightarrow C_{h}^{0}(\mathbb{Z}, X)$ is continuous.

Step 3 . The map $\mathbb{G}$ is completely continuous.

Set $V=\mathbb{G}\left(B_{R}\left(C_{h}^{0}(\mathbb{Z}, X)\right)\right), y=\mathbb{G} x$ for $x \in B_{R}\left(C_{h}^{0}(\mathbb{Z}, X)\right)$, where $R$ is a constant.

Firstly, we will prove that, for each $n \in \mathbb{Z}$, the set

$$
\Omega_{n}(V):=\left\{\frac{x(n)}{h(n)}: x \in V\right\}
$$

is relatively compact in $X$.

From condition (II), it follows that, for any $\varepsilon>0$, one can choose a constant $m \in \mathbb{Z}^{+}$s.t.

$$
\sum_{i=m}^{\infty}\left\|S_{\alpha}(i)\right\|(M(n-1-i) W(R h(n-1-i)) \leq \varepsilon .
$$

Since $y=\mathbb{G} x$ for $x \in B_{R}\left(C_{h}^{0}(\mathbb{Z}, X)\right)$,

$$
\begin{aligned}
y(n) & =\sum_{i=-\infty}^{n-1} S_{\alpha}(n-1-i) F(i, x(i)) \\
& =\sum_{i=0}^{+\infty} S_{\alpha}(i) F(n-1-i, x(n-1-i)) \\
& =\sum_{i=0}^{m-1} S_{\alpha}(i) F(n-1-i, x(n-1-i))+\sum_{k=m}^{\infty} S_{\alpha}(i) F(n-1-i, x(n-1-i)),
\end{aligned}
$$


we have

$$
\begin{aligned}
\frac{y(n)}{h(n)}= & \frac{m}{h(n)}\left(\frac{1}{m} \sum_{i=0}^{m-1} S_{\alpha}(i) F(n-1-i, x(n-1-i))\right) \\
& +\frac{1}{h(n)} \sum_{i=m}^{\infty} S_{\alpha}(i) F(n-1-i, x(n-1-i)) .
\end{aligned}
$$

Note that

$$
\begin{aligned}
& \frac{1}{h(n)}\left\|\sum_{i=m}^{\infty} S_{\alpha}(i) F(n-1-i, x(n-1-i))\right\| \\
& \quad \leq \frac{1}{h(n)} \sum_{i=m}^{\infty}\left\|S_{\alpha}(i)\right\| M((n-1-i)) W(\|x(n-1-i)\|) \\
& \quad \leq \frac{1}{h(n)} \sum_{i=m}^{\infty}\left\|S_{\alpha}(i)\right\| M((n-1-i)) W\left(\|x\|_{h} h(n-1-i)\right) \\
& \quad \leq \frac{1}{h(n)} \sum_{i=m}^{\infty}\left\|S_{\alpha}(i)\right\| M((n-1-i)) W(R h(n-1-i)) \leq \varepsilon,
\end{aligned}
$$

which implies

$$
\frac{y(n)}{h(n)} \in \frac{m}{h(n)} \overline{\operatorname{co}(S)}+B_{\varepsilon}(X),
$$

here $\overline{\operatorname{co}(S)}$ is the convex hull of $S$ and

$$
S=\bigcup_{i=0}^{m-1}\left\{S_{\alpha}(i) F(\tau, x): \tau \in[n-l, n-1] \cap \mathbb{Z},\|x\| \leq R_{1}\right\},
$$

with

$$
R_{1}=R \max _{\tau \in[n-l, n-1] \cap \mathbb{Z}} h(\tau) .
$$

It follows from $\left(\mathrm{H}_{0}\right)$ that $S$ is relatively compact in $X$, this together with

$$
\Omega_{n}(V) \subseteq \frac{m}{h(n)} \overline{\operatorname{co}(S)}+B_{\varepsilon}(X)
$$

shows that, for each $n \in \mathbb{Z}$, the set $\Omega_{n}(V)$ is relatively compact in $X$.

Then we show that the collection $V$ is weighted equiconvergent at $\pm \infty$.

For $x \in B_{R}\left(C_{h}^{0}(\mathbb{Z}, X)\right)$, one has

$$
\begin{aligned}
\frac{\|y(n)\|}{h(n)} & =\frac{1}{h(n)}\left\|\sum_{i=-\infty}^{n-1} S_{\alpha}(n-1-i) F(i, x(i))\right\| \\
& \leq \frac{1}{h(n)} \sum_{i=-\infty}^{n-1}\left\|S_{\alpha}(n-1-i)\right\| M(i) W(\|x(n)\|)
\end{aligned}
$$




$$
\leq \frac{1}{h(n)} \sum_{i=-\infty}^{n-1}\left\|S_{\alpha}(n-1-i)\right\| M(i) W\left(\|x\|_{h} h(i)\right)
$$

this together with condition (II) yields

$$
\lim _{|n| \rightarrow+\infty} \frac{\|y(n)\|}{h(n)}=0
$$

furthermore the convergence is independent of $x \in B_{R}\left(C_{h}^{0}(\mathbb{Z}, X)\right)$.

Then $V$ is a relatively compact set in $C_{h}^{0}(\mathbb{Z}, X)$ by Lemma 2.15 .

Step 4 . The set

$$
\left\{x \in C_{h}^{0}(\mathbb{Z}, X): x=\gamma \mathbb{G} x, \gamma \in(0,1)\right\}
$$

is bounded.

Let $x$ be a solution of the equation $x=\gamma \mathbb{G} x, \gamma \in(0,1)$ and $x(n) \in C_{h}^{0}(\mathbb{Z}, X)$. Compute $\|x\|_{h}$ as

$$
\begin{aligned}
\|x(n)\| & =\left\|\sum_{i=-\infty}^{n-1} S_{\alpha}(n-1-i) F(i, x(i))\right\| \\
& \leq \sum_{i=-\infty}^{n-1}\left\|S_{\alpha}(n-1-i)\right\| M(i) W(\|x(n)\|) \\
& \leq \sum_{i=-\infty}^{n-1}\left\|S_{\alpha}(n-1-i)\right\| M(i) W\left(\|x\|_{h} h(i)\right),
\end{aligned}
$$

which implies

$$
\frac{\|x(n)\|}{h(n)} \leq \sup _{n \in \mathbb{Z}}\left(\frac{1}{h(n)}\left(\sum_{i=-\infty}^{n-1}\left\|S_{\alpha}(n-1-i)\right\| M(i) W\left(\|x\|_{h} h(i)\right)\right)\right) \quad \text { for each } n \in \mathbb{Z} .
$$

Furthermore, one has the following estimate:

$$
\frac{\|x\|_{h}}{\sup _{n \in \mathbb{Z}}\left(\frac{1}{h(n)}\left(\sum_{i=-\infty}^{n-1}\left\|S_{\alpha}(n-1-i)\right\| M(i) W\left(\|x\|_{h} h(i)\right)\right)\right)} \leq 1 .
$$

According to condition (IV), we get the boundedness of the set (4.5).

Step 5. We claim that $\exists r_{0}>0$ s.t.

$$
\mathbb{G}\left(B_{r_{0}}\left(C_{h}^{0}(\mathbb{Z}, X)\right)\right) \subseteq B_{r_{0}}\left(C_{h}^{0}(\mathbb{Z}, X)\right) .
$$

If this is not true, then, for any $r>0$, one can find $x^{r} \in B_{r}\left(C_{h}^{0}(\mathbb{Z}, X)\right)$ such that $\left\|\mathbb{G} x^{r}\right\|_{h}>r$. Similar to the proof of Step 4, one can deduce that

$$
\frac{r}{\sup _{n \in \mathbb{Z}}\left(\frac{1}{h(n)}\left(\sum_{i=-\infty}^{n-1}\left\|S_{\alpha}(n-1-i)\right\| M(i) W(r h(i))\right)\right)} \leq 1,
$$


then

$$
\liminf _{r \rightarrow+\infty} \frac{r}{\sup _{n \in \mathbb{Z}}\left(\frac{1}{h(n)}\left(\sum_{i=-\infty}^{n-1}\left\|S_{\alpha}(n-1-i)\right\| M(i) W(r h(i))\right)\right)} \leq 1,
$$

which contradicts condition (IV) establishing the desired assertion.

Step 6. The map $\mathbb{G}$ has a fixed point.

From Lemma 4.3, together with Lemma 4.4, it follows that $F(k, x(k)) \in A A P(\mathbb{Z}, X)$ if $x \in A A P(\mathbb{Z}, X)$. Hence from Lemma 4.1, it follows that $\mathbb{G}(A A P(\mathbb{Z}, X)) \subseteq A A P(\mathbb{Z}, X)$. Consequently, combining with Step 5, we infer that

$$
\mathbb{G}\left(B_{r_{0}}\left(C_{h}^{0}(\mathbb{Z}, X)\right) \cap A A P(\mathbb{Z}, X)\right) \subseteq B_{r_{0}}\left(C_{h}^{0}(\mathbb{Z}, X)\right) \cap A A P(\mathbb{Z}, X) .
$$

Furthermore

$$
\begin{aligned}
\mathbb{G}\left(\overline{B_{r_{0}}\left(C_{h}^{0}(\mathbb{Z}, X)\right) \cap A A P(\mathbb{Z}, X)} C^{0}(\mathbb{Z}, X)\right. & \left.\subseteq \overline{\mathbb{G}\left(B_{r_{0}}\left(C_{h}^{0}(\mathbb{Z}, X)\right) \cap A A P(\mathbb{Z}, X)\right.} C_{h}^{0}(\mathbb{Z}, X)\right) \\
& \subseteq \overline{B_{r_{0}}\left(C_{h}^{0}(\mathbb{Z}, X)\right) \cap A A P(\mathbb{Z}, X)} C_{h}^{0}(\mathbb{Z}, X)
\end{aligned}
$$

here $\bar{S}^{C_{h}^{0}(\mathbb{Z}, X)}$ is the closure of $S$ in $C_{h}^{0}(\mathbb{Z}, X)$.

Consequently, we can consider

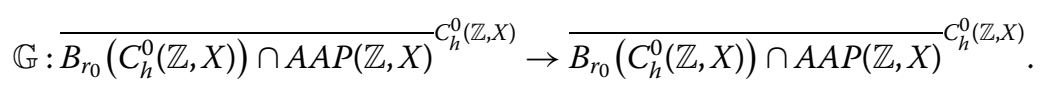

The arguments used in Steps 1-3 of this proof show that the map is completely continuous. From the result of Step 4 and Lemma 2.17 (the Leray-Schauder alternative theorem), it follows that $\mathbb{G}$ has a fixed point $x$ and

$$
x \in \overline{B_{r_{0}}\left(C_{h}^{0}(\mathbb{Z}, X)\right) \cap A A P(\mathbb{Z}, X)} C_{h}^{0}(\mathbb{Z}, X) .
$$

Step 7. The fixed point $x$ in Step 6 is discrete asymptotically almost periodic.

Let $\left(x_{n}\right)_{n}$ be a sequence in $B_{r_{0}}\left(C_{h}^{0}(\mathbb{Z}, X)\right) \cap A A P(\mathbb{Z}, X)$ such that $x_{n} \rightarrow x$, as $n \rightarrow \infty$ in the norm of $C_{h}^{0}(\mathbb{Z}, X)$. For $\varepsilon>0$, let $\delta>0$ be the constant in (III), one can select $n_{0} \in \mathbb{Z}^{+}$large enough so that

$$
\left\|x_{n}-x\right\|_{h} \leq \delta, \quad \text { for all } n \geq n_{0}
$$

From condition (III), it follows that, for $n \geq n_{0}$,

$$
\begin{aligned}
\left\|\mathbb{G} x_{n}-\mathbb{G} x\right\|_{d} & =\sup _{i \in \mathbb{Z}}\left\|\sum_{i=-\infty}^{n-1} S_{\alpha}(n-1-i)\left[F\left(i, x_{n}(i)\right)-F(i, x(i))\right]\right\| \\
& \leq \sup _{i \in \mathbb{Z}} \sum_{i=-\infty}^{n-1}\left\|S_{\alpha}(n-1-i)\right\|\left\|F\left(i, x_{n}(i)\right)-F(i, x(i))\right\| \leq \varepsilon,
\end{aligned}
$$

which means $\left(\mathbb{G} x_{n}\right)_{n}$ converges to $\mathbb{G} x=x$ uniformly in $\mathbb{Z}$, this together with $\mathbb{G} x_{n} \in$ $A A P(\mathbb{Z}, X)$, implies that $x \in A A P(\mathbb{Z}, X)$, and the proof is completed. 
If $F \in U C(\mathbb{Z} \times X, X)$, by Lemma 4.1, Lemma 4.2 and Lemma 2.17, we get the following result.

Theorem 4.5 Let h be given by Lemma 2.15, assume $\left(\mathrm{H}_{0}\right),\left(\mathrm{H}_{1}\right),\left(\mathrm{H}_{2}\right)$ hold, and F satisfies the following.

(I) There exists a nondecreasing and surjective function $W: \mathbb{R}^{+} \rightarrow \mathbb{R}^{+}$s.t.

$$
\|F(i, h(i) x)-F(i, h(i) y)\| \leq W(\|x-y\|), \quad \forall i \in \mathbb{Z}, x, y \in X
$$

(II) $\lim _{|n| \rightarrow+\infty} \frac{1}{h(n)} \sum_{i=-\infty}^{n-1}\left\|S_{\alpha}(n-1-i)\right\|\left(W\left(\|x\|_{h}\right)+\sup _{i \in \mathbb{Z}}\|F(i, 0)\|\right)=0$.

(III) $\liminf _{\tau \rightarrow+\infty} \frac{\tau}{\|S\|_{1}\left(W(\tau)+\sup _{i \in \mathbb{Z}}\|F(i, 0)\|\right)}>1$.

Then there exists a mild solution to Eq. (4.1) which is asymptotically almost periodic.

Proof Using the same symbols as Theorem 4.4, define a map $\mathbb{G}: C_{h}^{0}(\mathbb{Z}, X) \rightarrow C_{h}^{0}(\mathbb{Z}, X)$ by (4.4). For $x, y \in C_{h}^{0}(\mathbb{Z}, X)$, according to condition (I), one has the following estimates:

$$
\begin{aligned}
\|\mathbb{G} x(n)\| & \left\|\sum_{i=-\infty}^{n-1} S_{\alpha}(n-1-i) F(i, x(i))\right\| \\
\leq & \sum_{i=-\infty}^{n-1}\left\|S_{\alpha}(n-1-i)\right\|\|F(i, x(i))-F(i, 0)\|+\sum_{i=-\infty}^{n-1}\left\|S_{\alpha}(n-1-i)\right\|\|F(i, 0)\| \\
\leq & \sum_{i=-\infty}^{n-1}\left\|S_{\alpha}(n-1-i)\right\|\left(W\left(\|x\|_{h}\right)+\sup _{i \in \mathbb{Z}}\|F(i, 0)\|\right),
\end{aligned}
$$

which implies

$$
\frac{\|(\mathbb{G} x)(n)\|}{h(n)} \leq \frac{1}{h(n)} \sum_{i=-\infty}^{n-1}\left\|S_{\alpha}(n-1-i)\right\|\left(W\left(\|x\|_{h}\right)+\sup _{i \in \mathbb{Z}}\|F(i, 0)\|\right) .
$$

From condition (II), it follows that $\mathbb{G} x(n) \in C_{h}^{0}(\mathbb{Z}, X)$ and $\mathbb{G}$ is well defined.

Furthermore,

$$
\|F(i, x(i))-F(i, y(i))\| \leq W\left(\frac{\|x(i)-y(i)\|}{h(i)}\right) \leq W(\|x(i)-y(i)\|),
$$

which implies $F \in U C(\mathbb{Z} \times X, X)$.

For $x, y \in C_{h}^{0}(\mathbb{Z}, X)$, one has

$$
\begin{aligned}
\|(\mathbb{G} x)(n)-(\mathbb{G}) y(n)\| & =\left\|\sum_{i=-\infty}^{n-1} S_{\alpha}(n-1-i)[F(i, x(i))-F(i, y(i))]\right\| \\
& \leq \sum_{i=-\infty}^{n-1}\left\|S_{\alpha}(n-1-i)\right\|\|F(i, x(i))-F(i, y(i))\|
\end{aligned}
$$




$$
\begin{aligned}
& \leq \sum_{i=-\infty}^{n-1}\left\|S_{\alpha}(n-1-i)\right\| W\left(\frac{\|x(i)-y(i)\|}{h(i)}\right) \\
& \leq\left\|S_{\alpha}\right\|_{1} W\left(\|x-y\|_{h}\right),
\end{aligned}
$$

which implies that $\mathbb{G}$ is continuous.

Next, let $V=\mathbb{G}\left(B_{r}\left(C_{h}^{0}(\mathbb{Z}, X)\right)\right)$ and $y=\mathbb{G} x$ for $x \in B_{r}\left(C_{h}^{0}(\mathbb{Z}, X)\right)$, where $r$ is a constant.

Initially, we show that, for each $n \in \mathbb{Z}$, the set

$$
\Omega_{n}(V):=\left\{\frac{x(n)}{h(n)}: x \in V\right\}
$$

is relatively compact in $X$.

From condition (II), it follows that, for any $\varepsilon>0$, one can choose $m \in \mathbb{Z}^{+}$s.t.

$$
\sum_{i=m}^{\infty}\left\|S_{\alpha}(i)\right\|\left(W(r)+\sup _{i \in \mathbb{Z}}\|F(i, 0)\|\right) \leq \varepsilon .
$$

Since $y=\mathbb{G} x$ for $x \in B_{r}\left(C_{h}^{0}(\mathbb{Z}, X)\right)$,

$$
\begin{aligned}
y(n) & =\sum_{i=-\infty}^{n-1} S_{\alpha}(n-1-i) F(i, x(i)) \\
& =\sum_{i=0}^{+\infty} S_{\alpha}(i) F(n-1-i, x(n-1-i)) \\
& =\sum_{i=0}^{m-1} S_{\alpha}(i) F(n-1-i, x(n-1-i))+\sum_{i=m}^{\infty} S_{\alpha}(i) F(n-1-i, x(n-1-i)),
\end{aligned}
$$

we have

$$
\begin{aligned}
\frac{y(n)}{h(n)}= & \frac{m}{h(n)}\left(\frac{1}{m} \sum_{i=0}^{m-1} S_{\alpha}(i) F(n-1-i, x(n-1-i))\right) \\
& +\frac{1}{h(n)} \sum_{i=m}^{\infty} S_{\alpha}(i) F(n-1-i, x(n-1-i)) .
\end{aligned}
$$

Note that

$$
\begin{aligned}
& \frac{1}{h(n)}\left\|\sum_{i=m}^{\infty} S_{\alpha}(i) F(n-1-i, x(n-1-i))\right\| \\
& \leq \frac{1}{h(n)} \sum_{i=m}^{\infty}\left\|S_{\alpha}(i)\right\|\|F(n-1-i, x(n-1-i))-F(n-1-i, 0)\| \\
& \quad+\sum_{i=m}^{\infty}\left\|S_{\alpha}(i)\right\|\|F(n-1-i, 0)\|
\end{aligned}
$$




$$
\begin{aligned}
& \leq \frac{1}{h(n)} \sum_{i=m}^{\infty}\left\|S_{\alpha}(i)\right\| W\left(\|x\|_{h}\right)+\sum_{i=m}^{\infty}\left\|S_{\alpha}(i)\right\| \sup _{i \in \mathbb{Z}}\|F(i, 0)\| \\
& \leq \frac{1}{h(n)} \sum_{i=m}^{\infty}\left\|S_{\alpha}(i)\right\|\left(W(r)+\sup _{i \in \mathbb{Z}}\|F(i, 0)\|\right) \leq \varepsilon,
\end{aligned}
$$

which implies

$$
\frac{y(n)}{h(n)} \in \frac{m}{h(n)} \overline{\operatorname{co}(S)}+B_{\varepsilon}(X),
$$

here $\overline{\operatorname{co}(S)}$ is the convex hull of $S$ and

$$
S=\bigcup_{k=0}^{l-1}\left\{S_{\alpha}(k) F(\tau, x): \tau \in[n-l, n-1] \cap \mathbb{Z},\|x\| \leq r_{1}\right\},
$$

with

$$
r_{1}=r \max _{\tau \in[n-l, n-1] \cap \mathbb{Z}} h(\tau) .
$$

It follows from $\left(\mathrm{H}_{0}\right)$ that $S$ is relatively compact in $X$, this, together with

$$
\Omega_{n}(V) \subseteq \frac{m}{h(n)} \overline{\operatorname{co}(S)}+B_{\varepsilon}(X)
$$

shows that, for all $n \in \mathbb{Z}$, the set $\Omega_{n}(V)$ is relatively compact in $X$.

Then we show the map $\mathbb{G}$ is completely continuous.

Let $x \in B_{r}\left(C_{h}^{0}(\mathbb{Z}, X)\right)$, one has

$$
\begin{aligned}
& \frac{\|y(n)\|}{h(n)} \\
& =\frac{1}{h(n)}\left\|\sum_{k=-\infty}^{n-1} S_{\alpha}(n-1-k) F(k, x(k))\right\| \\
& \leq \frac{1}{h(n)}\left[\sum_{k=-\infty}^{n-1}\left\|S_{\alpha}(n-1-k)\right\|\|F(k, x(k))-F(k, 0)\|+\sum_{k=-\infty}^{n-1}\left\|S_{\alpha}(n-1-k)\right\|\|F(k, 0)\|\right] \\
& \leq \frac{1}{h(n)}\left[\sum_{k=-\infty}^{n-1}\left\|S_{\alpha}(n-1-k)\right\|\left(W\left(\|x\|_{h}\right)+\sup _{k \in \mathbb{Z}}\|F(k, 0)\|\right)\right] \\
& \leq \frac{1}{h(n)} \sum_{k=-\infty}^{n-1}\left\|S_{\alpha}(n-1-k)\right\|\left(W\left(\|x\|_{h}\right)+\sup _{k \in \mathbb{Z}}\|F(k, 0)\|\right),
\end{aligned}
$$

this together with condition (II) yields

$$
\lim _{|n| \rightarrow+\infty} \frac{\|y(n)\|}{h(n)}=0
$$

uniform in $x \in B_{r}\left(C_{h}^{0}(\mathbb{Z}, X)\right)$. By Lemma $2.15, \mathbb{G}$ is completely continuous. 
Finally, we prove that the collection

$$
\left\{x \in C_{h}^{0}(\mathbb{Z}, X): x=\gamma \mathbb{G} x, \gamma \in(0,1)\right\}
$$

is bounded.

If $x \in C_{h}^{0}(\mathbb{Z}, X)$ is a solution of $x=\gamma \mathbb{G} x$ for $0<\gamma<1$, then similar to Step 4 of the proof of Theorem 4.4, one has

$$
\frac{\|x\|_{h}}{\left\|S_{\alpha}\right\|_{1}\left(W\left(\|x\|_{h}\right)+\sup _{k \in \mathbb{Z}}\|F(k, 0)\|\right)} \leq 1 .
$$

According to condition (III), we get the boundedness of the set (4.6).

Finally, similar to the proof of Theorem 4.4, together with Lemma 4.1 and Lemma 4.2, there is a mild solution to Eq. (4.1), which is asymptotically almost periodic by Lemma 2.17 .

\section{Applications}

In this section, an example is provided to demonstrate the effectiveness of our abstract results.

Consider the difference equation of fractional order of the following form:

$$
\triangle^{\alpha} x(n)=A x(n+1)+\frac{\mu g(n)[1+x(n)]}{1+\|x\|_{d}}, \quad n \in \mathbb{Z},
$$

where $\alpha \in(0,1)$, the $C_{0}$-semigroup on $X$ generated by $A$ satisfies (3.2), $g(n): \mathbb{Z} \rightarrow X$ is an asymptotically almost periodic sequence, and $\mu>0$ is a parameter.

It follows from Lemma 3.1 that there exists a discrete $\alpha$-resolvent family $\left\{S_{\alpha}(n)\right\}_{n \in \mathbb{N}}$ generated by $A$, which is summable and

$$
\left\|S_{\alpha}\right\|_{1} \leq \frac{1}{\delta}
$$

Thus $\left(\mathrm{H}_{1}\right)$ holds.

Let

$$
g(n):=\sin n+\sin \sqrt{2} n+e^{-|n|} .
$$

Thus from Lemma 2.10, it follows that $g: \mathbb{Z} \times \mathbb{R} \rightarrow \mathbb{R}, g(n) \in A A P(\mathbb{Z} \times \mathbb{R}, \mathbb{R})$. Furthermore

$$
F(n, x)=\frac{\mu g(n)[1+x(n)]}{1+\|x\|_{d}}, \quad n \in \mathbb{N}, x \in X,
$$

belongs to $A A P(\mathbb{Z} \times X, X)$ and it is bounded, thus $\left(\mathrm{H}_{2}\right)$ holds. Moreover, it is clear that $\left(\mathrm{H}_{0}\right)$ holds.

On the other hand,

$$
\|F(n, x)-F(n, y)\|_{d} \leq \mu\|g\|_{d}\left\|\frac{1+x(n)}{1+\|x\|_{d}}-\frac{1+y(n)]}{1+\|y\|_{d}}\right\| \leq 6 \mu\|x-y\|_{d}, \quad n \in \mathbb{Z}, x, y \in X,
$$


that is, the assumptions $\left(\mathrm{H}_{3}\right)$ holds with $L_{F}=6 \mu$. Then from Corollary 4.5 it follows that there exists a unique mild solution to Eq. (5.1) whenever $6 \mu<\delta$, moreover, it is asymptotically almost periodic.

\section{Funding}

The work was supported by the Characteristic Innovation Project (Natural Science) of Guangdong Province (2016KTSCX094), the Science and Technology Program Project of Guangzhou (201707010230), National Natural Science Foundation of China $(11871225,11671339)$. The second author extends his appreciation to the Deanship of Scientific Research at King Saud University for funding this work through research group no. RGP-237.

\section{Availability of data and materials}

Not applicable.

\section{Competing interests}

The authors declare that they have no competing interests.

\section{Authors' contributions}

Each of the authors, JC, MS and YZ contributed equally to each part of this work. All authors read and approved the final manuscript.

\section{Author details}

${ }^{1}$ Department of Mathematics, Guangdong University of Education, Guangzhou, P.R. China. ${ }^{2}$ Department of Mathematics, College of Science, King Saud University, Riyadh, Saudi Arabia. ${ }^{3}$ Faculty of Mathematics and Computational Science, Xiangtan University, Xiangtan, P.R. China. ${ }^{4}$ Nonlinear Analysis and Applied Mathematics (NAAM) Research Group, Faculty of Science, King Abdulaziz University, Jeddah, Saudi Arabia.

\section{Publisher's Note}

Springer Nature remains neutral with regard to jurisdictional claims in published maps and institutional affiliations.

Received: 6 May 2019 Accepted: 26 August 2019 Published online: 02 September 2019

\section{References}

1. Agarwal, R.: Difference Equations and Inequalities. Dekker, New York (1992)

2. Murty, K., Anand, P., Lakshimiprasannam, V.: First order difference system-existence and uniqueness. Proc. Am. Math. Soc. 125, 3533-3539 (1997)

3. Kurzweil, J., Papsschinopoulce, G.: Topological equivalence and structural stability for linear difference equations. J. Differ. Equ. 89, 89-94 (1991)

4. Walther, A.: Fastperiodische Folgen und Potenzreihen mit fastperiodischen Koeffzienten. Abh. Math. Semin. Univ. Hamb. 6, 217-234 (1928)

5. Walther, A.: Fastperiodische folgen und ihre fouriersche analysis. Atti Congr. Int. Math. 2, 289-298 (1928)

6. Halanay, A.: Solutions périodiques et presque-périodiques des systémes d'équations aux différences finies. Arch. Ration. Mech. Anal. 12,134-149 (1963)

7. Corduneanu, C.: Almost periodic discrete processes. Libertas Math. 2, 159-169 (1982)

8. Fréchet, M.: Les fonctions asymptotiquement presque-périodiques continues. C. R. Acad. Sci. Paris 213, 520-522 (1941) (in French)

9. Fréchet, M.: Les fonctions asymptotiquement presque-périodiques. Rev. Sci. (Rev. Rose. Illus.) 79, 341-354 (1941) (in French)

10. Fan, K.: Les fonctions asymptotiquement presque-périodiques d'une variable entière et leur application à l'étude de l'itération des transformations continues. Math. Z. 48, 685-711 (1943)

11. Zhang, S., Liu, P., Gopalsamy, K.: Almost periodic solutions of nonautonomous linear difference equations. Appl. Anal. 81, 281-301 (2002)

12. Thanh, N.: Asymptotically almost periodic solutions on the half-line. J. Differ. Equ. Appl. 11, 1231-1243 (2005)

13. Song, Y.: Asymptotically almost periodic solutions of nonlinear Volterra difference equations with unbounded delay. J. Differ. Equ. Appl. 14, 971-986 (2008)

14. Campo, L., Pinto, M., Vidal, C.: Almost and asymptotically almost periodic solutions of abstract retarded functional difference equations in phase space. J. Differ. Equ. Appl. 17, 915-934 (2011)

15. Caraballo, T., Cheban, D.: Almost periodic motions in semi-group dynamical systems and Bohr/Levitan almost periodic solutions of linear difference equations without Favard's separation condition. J. Differ. Equ. Appl. 19, 872-897 (2013)

16. Cuevas, C., Dantas, F., Soto, H.: Almost periodicity for a nonautonomous discrete dispersive population model. Numer Funct. Anal. Optim. 37, 1503-1516 (2016)

17. Zhou, Y.: Attractivity for fractional differential equations in Banach space. Appl. Math. Lett. 75, 1-6 (2018)

18. Zhou, Y.: Attractivity for fractional evolution equations with almost sectorial operators. Fract. Calc. Appl. Anal. 21(3), 786-800 (2018)

19. Zhou, Y., Shangerganesh, L., Manimaran, J., Debbouche, A.: A class of time-fractional reaction-diffusion equation with nonlocal boundary condition. Math. Methods Appl. Sci. 41, 2987-2999 (2018)

20. Zhou, Y., Peng, L., Huang, Y.Q.: Duhamel's formula for time-fractional Schrödinger equations. Math. Methods Appl. Sci. 41, 8345-8349 (2018) 
21. Zhou, Y., Peng, L., Huang, Y.Q.: Existence and Hölder continuity of solutions for time-fractional Navier-Stokes equations. Math. Methods Appl. Sci. 41, 7830-7838 (2018)

22. Atici, F., Sengül, S.: Modeling with fractional difference equations. J. Math. Anal. Appl. 369, 1-9 (2010)

23. Goodrich, C.: Existence and uniqueness of solutions to a fractional difference equation with nonlocal conditions. Comput. Math. Appl. 61, 191-202 (2011)

24. Goodrich, C: Existence of a positive solution to a system of discrete fractional boundary value problems. Appl. Math. Comput. 217, 4740-4753 (2011)

25. Dahal, R., Goodrich, C.: A monotonicity result for discrete fractional difference operators. Arch. Math. (Basel) 102 293-299 (2014)

26. Wu, G., Baleanu, D.: Discrete fractional logistic map and its chaos. Nonlinear Dyn. 75, 283-287 (2014)

27. Lizama, C.: The Poisson distribution, abstract fractional difference equations, and stability. Proc. Am. Math. Soc. 145 3809-3827 (2017)

28. Abadias, L., Lizama, C.: Almost automorphic mild solutions to fractional partial difference-differential equations. Appl. Anal. 95, 1347-1369 (2016)

29. Corduneanu, C.: Almost Periodic Functions. Wiley, New York (1968)

30. Zhang, S.: Almost periodic solutions of difference equations. Chin. Sci. Bull. 43, 2041-2047 (1998)

31. Zhang, S.: Existence of almost periodic solution for difference systems. Ann. Differ. Equ. 43, 184-206 (2000)

32. Long, W., Pan, W.: Asymptotically almost periodic solution to a class of Volterra difference equations. Adv. Differ. Equ. 2012, 199 (2012)

33. Gohberg, I., Feldman, I.: Convolution Equations and Projection Methods for Their Solution. AMS Translation of Mathematical Monographs. Am. Math. Soc., Providence (1974)

34. Cuevas, C., Pinto, M.: Convergent solutions of linear functional difference equations in phase space. J. Math. Anal. Appl. 277, 324-341 (2003)

35. Agarwal, R., Cuevas, C., Dantas, F.: Almost automorphy profile of solutions for difference equations of Volterra type. J. Appl. Math. Comput. 42, 1-18 (2013)

36. Matkowski, J.: Integrable solutions of functional equations. Diss. Math. 127, 1-68 (1975)

37. Granas, A., Dugundji, J.: Fixed Point Theory. Springer, New York (2003)

\section{Submit your manuscript to a SpringerOpen ${ }^{\circ}$ journal and benefit from:}

- Convenient online submission

- Rigorous peer review

- Open access: articles freely available online

- High visibility within the field

- Retaining the copyright to your article

Submit your next manuscript at $\boldsymbol{~ s p r i n g e r o p e n . c o m ~}$ 